\title{
Liver macrophages inhibit the endogenous antioxidant response in obesity-associated insulin resistance
}

\author{
Valerio Azzimato ${ }^{1 *}$, Jennifer Jager ${ }^{2 *}$, Ping Chen ${ }^{1 *}$, Cecilia Morgantini ${ }^{1}$, Laura Levi ${ }^{1}$, \\ Emelie Barreby ${ }^{1}$, André Sulen ${ }^{1}$, Carolina Oses ${ }^{1}$, Joost Willerbrords ${ }^{1}$, Connie $\mathrm{Xu}^{1}{ }^{1}, \mathrm{Xidan} \mathrm{Li}^{1}$, \\ Joanne X. Shen ${ }^{3}$, Naveed Akbar ${ }^{4}$, Lars Haag ${ }^{5}$, Ewa Ellis ${ }^{6}$, Kerstin Wålhen ${ }^{7}$, Erik Näslund ${ }^{8}$, \\ Anders Thorell ${ }^{8,9}$, Robin P. Choudhury ${ }^{4}$, Volker M. Lauschke ${ }^{3}$, Mikael Rydén ${ }^{7}$, \\ Siobhan M. Craige ${ }^{10}$, Myriam Aouadi ${ }^{1 \dagger}$
}

Obesity and insulin resistance are risk factors for nonalcoholic fatty liver disease (NAFLD), the most common chronic liver disease worldwide. Because no approved medication nor an accurate and noninvasive diagnosis is currently available for NAFLD, there is a clear need to better understand the link between obesity and NAFLD. Lipid accumulation during obesity is known to be associated with oxidative stress and inflammatory activation of liver macrophages (LMs). However, we show that although LMs do not become proinflammatory during obesity, they display signs of oxidative stress. In livers of both humans and mice, antioxidant nuclear factor erythroid 2related factor 2 (NRF2) was down-regulated with obesity and insulin resistance, yielding an impaired response to lipid accumulation. At the molecular level, a microRNA-targeting NRF2 protein, miR-144, was elevated in the livers of obese insulin-resistant humans and mice, and specific silencing of miR-144 in murine and human LMs was sufficient to restore NRF2 protein expression and the antioxidant response. These results highlight the pathological role of LMs and their therapeutic potential to restore the impaired endogenous antioxidant response in obesity-associated NAFLD.

\section{INTRODUCTION}

Obesity represents a major health issue worldwide as it strongly increases the risk for several metabolic complications including nonalcoholic fatty liver disease (NAFLD), insulin resistance, and type 2 diabetes $(1,2)$. Given its major role in the metabolism of nutrients, the liver plays a central role in the control of metabolic homeostasis (3).

Fatty liver is the result of excessive lipid accumulation due to the lower fat storage capacity of adipose tissue in obesity-associated insulin resistance (4). The inability of the liver to handle this overload of fat leads to aberrant lipid peroxidation and excessive production of reactive oxygen species (ROS) and reactive nitrogen species (RNS) (5). ROS and RNS are thought to trigger the phenotypic switch of liver macrophages (LMs) from an anti-inflammatory (M2) to a proinflammatory activation state (M1), leading to insulin resistance (6). The contribution of LMs to oxidative stress in the liver has been debated, and despite several reports of LM activation leading to unbalanced and detrimental ROS production in liver diseases (7), the direct role of LMs in the regulation of oxidative stress during the

\footnotetext{
'Integrated Cardio Metabolic Center, Department of Medicine, Karolinska Institutet, 14157 Huddinge, Sweden. ${ }^{2}$ Université Côte d'Azur, Inserm U1065, C3M, Team Cellula and Molecular Physiopathology of Obesity, 06000 Nice, France. ${ }^{3}$ Section of Pharmacogenetics, Department of Physiology and Pharmacology, Karolinska Institutet, 17177 Solna, Sweden. ${ }^{4}$ Division of Cardiovascular Medicine, Radcliffe Department of Medicine, University of Oxford, OX3 9DU Oxford, UK. ${ }^{5}$ Department of Laboratory Medicine, Laboratory Medicine, Karolinska Institutet, 14157 Huddinge, Sweden. ${ }^{6}$ Division of Transplantation Surgery, Clinical Science, Intervention and Technology (CLINTEC), Karolinska Institutet, 14157 Huddinge, Sweden. ${ }^{7}$ Unit of Endocrinology Department of Medicine, Karolinska Institutet, 14157 Huddinge, Sweden. ${ }^{8}$ Division of Surgery, Department of Clinical Sciences, Danderyd Hospital, Karolinska Institutet, 18288 Stockholm, Sweden. ${ }^{9}$ Department of Surgery, Ersta Hospital, Karolinska Institutet, 11628 Stockholm, Sweden. ${ }^{10}$ Human Nutrition, Food, and Exercise Department, Virginia Tech, Blacksburg, VA 24060, USA.

*These authors contributed equally to this study.

tCorresponding author. Email: myriam.aouadi@ki.se
}

initial disease state was unknown. Studies have described macrophages and particularly LMs as the major source of ROS (8), primarily referred to proinflammatory-activated macrophages (9). However, we recently demonstrated that LMs do not undergo proinflammatory activation with obesity or insulin resistance in mice or humans (10). We have also demonstrated that, during obesity, LMs produce noninflammatory factors able to regulate insulin sensitivity but nonetheless display signs of oxidative stress. Transcriptomic profiling showed that several metabolic pathways involved in ROS/RNS production such as the tricarboxylic acid cycle (TCA) cycle and oxidative phosphorylation were dysregulated in the LMs of obese compared to control mice (10). We therefore hypothesized that LMs may regulate oxidative stress independently of their inflammatory status in obesityinduced insulin resistance.

Nuclear factor erythroid 2-related factor 2 (NFE2L2/NRF2), a basic leucine zipper transcription factor, is a master regulator of redox homeostasis (11). Under normal physiological conditions, NRF2 is targeted for proteasomal degradation through its association with Kelch-like ECH-associated protein-1 (KEAP1). Conversely, upon oxidative stress, this complex dissociates, and NRF2 translocates to the nucleus where it binds to the antioxidant responsive element (ARE), thereby driving the antioxidant response. The antioxidant capacity of the liver is reduced in obesity, although the molecular mechanisms underlying this impairment remain unknown (12). MicroRNAs (miRNAs) are short, single-stranded noncoding RNAs of about 21 to 23 nucleotides in length (13) that bind to target mRNAs at the $3^{\prime}$ untranslated region and exert their function through mRNA degradation or protein translation inhibition (14). We demonstrate that, in mouse models of obesity-induced insulin resistance characterized by excessive hepatic lipid accumulation and in human obese individuals, LMs produce miRNAs that impair the antioxidant capacity of the liver but are not linked to activation of proinflammatory pathways. 


\section{RESULTS}

\section{Oxidative stress in LMs fails to trigger an antioxidant response in obesity-induced insulin resistance}

Increased lipid peroxidation products are a common marker of oxidative stress (15). To confirm that high-fat diet (HFD)-induced obesity is associated with increased lipid peroxidation in the liver, we measured concentrations of malondialdehyde (MDA), a reactive aldehyde produced during lipid peroxidation, in livers of obese $o b / o b$ mice. As expected, mice fed an HFD for 9 weeks showed a significant increase in weight and impaired glucose handling $(P<0.0001$; fig. S1, A and B) coupled with increased hepatic lipid and MDA accumulation $(P<0.01$; Fig. $1, A$ and $B)$. In addition, ROS release in media cultured with LMs isolated from obese HFD mice was increased compared to normal diet controls (Fig. 1C). Intracellular RNS concentrations also increased in the isolated LMs (Fig. 1D). Moreover, treatment of obesity-associated LMs with superoxide dismutase, catalase, or the inhibitor of nitric oxide synthase activity $\mathrm{N}(\omega)$-nitro-Larginine methyl ester highlighted that both ROS and RNS contribute to oxidative stress in LMs during obesity (fig. S1, C to F).

To study the phenotype of LMs in obesity, we analyzed the transcriptomic profiles of obese $o b / o b$ mice at two different ages ( 9 and 14 weeks) compared to age-matched wild-type (wt) lean controls. Gene Ontology (GO) enrichment analysis revealed oxidative stress as one of the most dysregulated biological processes in obesity (Fig. 1D and tables S1 and S2 in data file S1). In addition, pathway analysis demonstrated impairment of lipid oxidation and antioxidant response pathways in obese compared with lean mice (fig. S1G, complete list in tables S3 and S4). We observed dissimilar findings in the LMs of mice fed an HFD for 9 weeks, where pathways involved in oxidative stress were enriched compared to normal diet (ND) controls (fig. S1, $\mathrm{H}$ and $\mathrm{I}$, and tables $\mathrm{S} 5$ and $\mathrm{S6}$ ). Consistent with our previous findings (10), transcriptomic profiling failed to reveal a proinflammatory phenotypic switch in LMs in obese compared to ND mice (fig. S1J and tables S7 and S8).

Because oxidative stress is known to trigger the endogenous antioxidant response under the control of the transcription factor $N R F 2$, we measured NRF2 expression in LMs from $o b / o b$ mice. We observed that mRNA expression of Nrf2 was not changed in LMs of obese mice compared to lean mice (Fig. 1E). As RNA sequencing (RNA-seq) only measures steady-state transcript abundance, we also performed global run-on sequencing (GRO-seq), which allows the measurement of nascent transcripts. This revealed that Nrf2 transcription remained unchanged in HFD mice (Fig. 1F). RNA-seq and GRO-seq analyses indicated that expression of most NRF2 target genes remained unchanged in obesity despite the increased ROS in LMs (Fig. 1, H and I, and table S9). However, we observed a decrease in NRF2 protein expression in the LMs of HFD-fed mice compared to controls (Fig. 1J). NRF2 was also decreased in hepatocytes and whole livers of HFD-fed mice compared to ND controls (Fig. 1, K and L) and in livers of $o b / o b$ mice compared to wt (Fig. 1L). Therefore, the antioxidant response driven by NRF2 is impaired in LMs and hepatocytes in both HFD- and genetically induced obesity.

To test whether the impaired antioxidant response observed in the livers from obese mice also occurred in humans, we measured oxidative stress and NRF2 mRNA and protein in lean, obese insulinsensitive, and obese insulin-resistant individuals (Table 1). Lipid peroxidation was increased in obese insulin-sensitive compared to lean individuals, and further increased in insulin-resistant obesity (Fig. 1M). We also observed higher intracellular ROS and RNS ac- cumulation in the livers of obese compared to lean individuals (fig. S1K). These ROS and RNS were exacerbated in insulin resistance and associated with liver oxidative stress, obesity, and insulin resistance. As in mice, we observed no change in human NRF2 mRNA (Fig. $1 \mathrm{~N}$ ), but NRF2 protein expression was greatly decreased in obese insulin-resistant individuals compared to obese insulin-sensitive or lean individuals (Fig. 1O). Together, these results demonstrate that oxidative stress fails to induce an NRF2-mediated antioxidant response during obesity-associated insulin resistance in mouse and human livers.

\section{NRF2 is a target of $\boldsymbol{m i R - 1 4 4}$ in obesity-associated LMs}

We next investigated the mechanism(s) whereby NRF2 protein abundance decreased with insulin resistance. Because transcription of NRF2 is unaffected by obesity, and KEAP1 is an important regulator of NRF2 through its ubiquitination and degradation via the proteasome (16), we investigated the KEAP1-NRF2 interaction in liver samples from obese insulin-resistant, obese insulin-sensitive, and lean individuals. Although expression of KEAP1 was unaltered in the livers from obese insulin-resistant and insulin-sensitive individuals, the amount of NRF2 associated with KEAP1 was reduced in obesity-associated insulin resistance (Fig. 2A). However, this result could potentially be due to the overall down-regulation of NRF2 rather than a decreased interaction between NRF2/KEAP1. Moreover, NRF2 ubiquitination remained unchanged in humans (fig. S2A). These data suggested that the decrease in NRF2 was may be due not only to ubiquitination but was also to an alternative posttranscriptional mechanism independent of KEAP1-induced degradation. Given that NRF2 was down-regulated in LMs of obese mice, we analyzed the LM miRNome in diet-induced obese mice to investigate whether miRNAs could regulate NRF2 posttranscriptionally. We performed small RNA-seq on LMs of 9- and 14-week-old ob/ob mice and found that six miRNAs were significantly and commonly up-regulated in the obese mice compared to age-matched wt controls $(P<0.05$; Fig. 2, B and C). Using a predictive in silico database (17), we noted that NRF2 was an experimentally validated target of $m i R-144$, one of the up-regulated miRNAs.

We therefore performed stem-loop reverse transcription quantitative polymerase chain reaction (RT-qPCR) analysis on LMs from both the HFD and $o b / o b$ obesity models. We found that $m i R-144$ expression increased in LMs isolated from HFD mice (Fig. 2D) and in the livers of both HFD-fed and $o b / o b$ mice compared to their respective controls (Fig. 2E). The observed increase of $m i R-144$ was liver-specific as its expression remained unchanged in the spleen, lung, and visceral adipose tissue of mice fed an HFD (fig. S2, B to D). Furthermore, $m i R-144$ expression was significantly increased in the livers of obese insulin-resistant compared to obese insulin-sensitive or lean individuals $(P<0.01, P<0.0001$, and $P<0.001$, respectively; Fig. $2 \mathrm{~F})$. Together, these data suggest that $m i R-144$ may mediate the decrease in NRF2 in obesity-associated insulin resistance in both murine and human livers.

\section{The transcription factor GATA4 drives the expression of $\mathbf{m i R - 1 4 4}$ in the liver of insulin-resistant individuals}

To investigate the mechanism triggering the increase of $m i R-144$ in insulin resistance, we performed targeted in silico analysis of the $m i R-144$ promoter region. We identified a high density of GATA binding protein 4 (GATA4)-binding domains on the $m i R-144$ promoter and enhancer regions (Fig. $3 \mathrm{~A}$ ), which prompted us to analyze whether the 
A

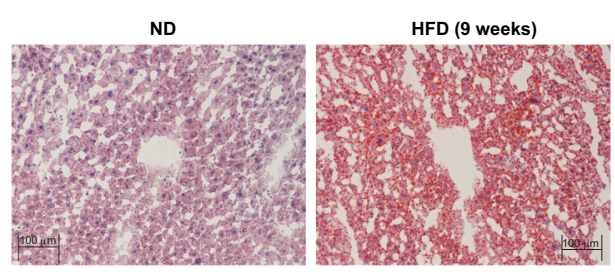

E
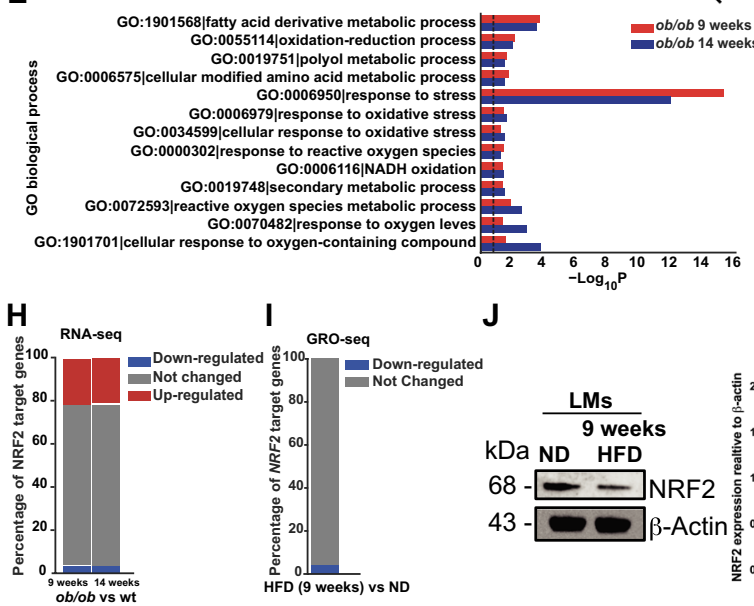

I GRO-seq

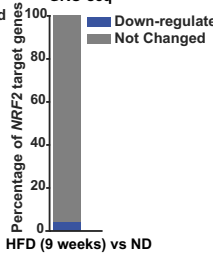

J

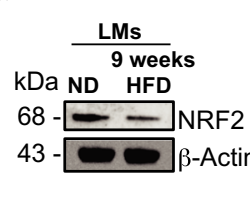

B

C
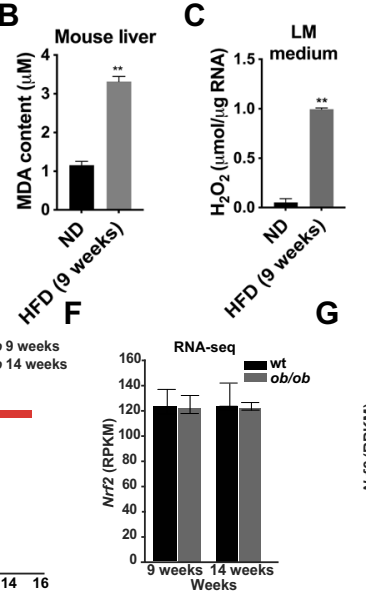

K
L

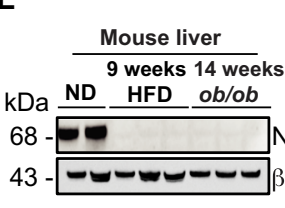

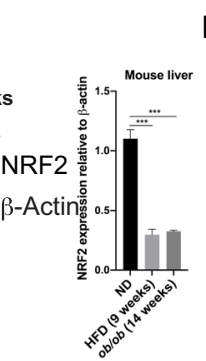

M

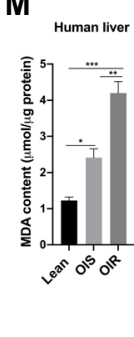

$\mathbf{N}$

Human liver

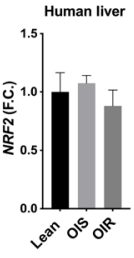

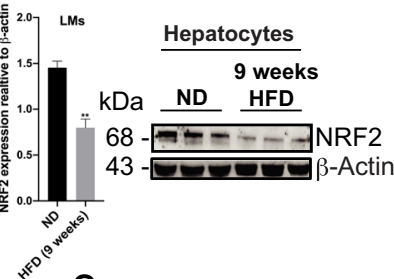

0

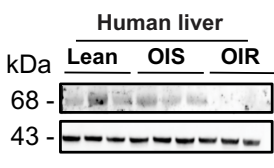

Fig. 1. Oxidative stress in LMs fails to trigger an antioxidant response in obesity-induced insulin resistance. (A and B) Liver Oil Red O staining (A) and MDA content (B) of mice fed an HFD or ND for 9 weeks ( $n=4$ per group). (C) Extracellular $\mathrm{H}_{2} \mathrm{O}_{2}$ content in media from LMs of mice fed an HFD or ND for 9 weeks ( $n=4$ per condition). (D) Intracellular RNS content in LMs of mice fed an HFD or ND for 9 weeks ( $n=3$ per condition). RFU, relative fluorescence units. (E) Selected significantly enriched Gene Ontology (GO) biological process terms from genes differentially expressed in LMs of 9- and 14-week-old ob/ob mice compared to wt mice ( $n=4 \mathrm{wt}, n=3 o b / o b$ for 9 weeks; $n=4$ for 14 weeks). NADH, reduced form of nicotinamide adenine dinucleotide. (F and $\mathbf{G}$ ) Nrf2 expression data from (F) RNA-seq of 9- and 14-week-old wt and ob/ob mice and (G) GRO-seq of mice fed an HFD or ND for 9 weeks ( $n=4$ wt, $n=3$ ob/ob, ND, or HFD for 9 weeks; $n=4$ for 14 weeks). (H and I) Percentage of NRF2 target genes significantly up-regulated, down-regulated, or without differential expression in (H) RNA-seq of 9- and 14-week-old ob/ob mice compared to age-matched wt mice and (I) GRO-seq of mice fed an HFD or ND for 9 weeks ( $n=4 \mathrm{wt}$, ND, or HFD, $n=3$ ob/ob for 9 weeks; $n=4$ for 14 weeks; $P<0.05$, $0.2<\mid \log _{2}$ fold change $>0.2$ ). ( $\mathbf{J}$ to $\mathbf{L}$ ) Western blots for NRF2 in LMs (J), hepatocytes (K), and whole livers (L) from 9-and 14-week-old $o b / o b$ mice fed an HFD or ND for 9 weeks ( $n=3$ per condition). (M) Liver MDA content from lean, obese insulin-sensitive (OIS), and obese insulin-resistant (OIR) human individuals ( $n=5$ per condition). (N) RT-qPCR analysis of Nrf2 in liver biopsies from lean, OIS, and OIR human individuals ( $n=5$ per condition). Fold change (F.C.) calculation compared to lean. (O) Western blots of NRF2 in the livers from lean, OIS, and OIR human individuals ( $n=5$ per condition). Data are means \pm SEM. ${ }^{*} P<0.05,{ }^{* *} P<0.01,{ }^{* *} P<0.001$, and ${ }^{* * *} P<0.0001$. RPKM, reads per kilobase of transcript, per million mapped reads.

expression or phosphorylation of the GATA4 isoform were altered in insulin resistance. In liver protein lysates from obese insulin-resistant participants, both GATA4 protein expression and phosphorylation were significantly higher than in lysates from obese insulin-sensitive and lean individuals $(P<0.05$ and $P<0.01$, respectively; Fig. 3B). To test the hypothesis that GATA4 induces the expression of miR-144 in insulin resistance, we performed chromatin immunoprecipitation
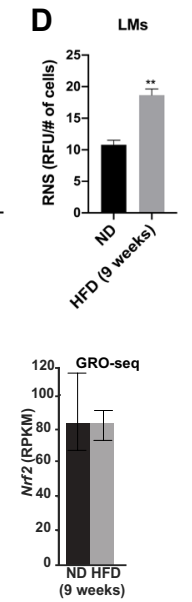

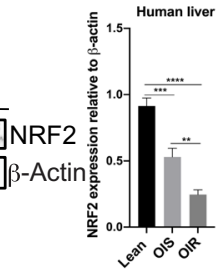

(ChIP) to analyze specific binding of GATA4 to the miR-144 promoter region. ChIP analysis confirmed that GATA4 increased binding to the $m i R-144$ promoter in obese insulin-resistant compared to lean individuals (Fig. 3C). We also observed greater amounts in trimethylation of lysine 4 of the histone 3 (H3K4me3) modification, a well-known marker for active transcription, in the insulin-resistant condition (Fig. 3D), suggesting transcription of the miR-144 locus.

Because oxidative stress is known to activate the extracellular signal-regulated kinase $(E R K)$ pathway and lead to the activation of GATA4 by phosphorylation (18), we measured ERK1/2 activity in the livers from our human cohort. We observed that ERK1/2 phosphorylation increased with obesity but to similar degrees as in insulin-resistant and insulinsensitive individuals (Fig. 3E).

We then used the ob/ob-Erk1 ${ }^{-1-}$ mouse model (19) to validate the role of the ERK pathway in the regulation of GATA4 activity in obesity. Western blot analysis showed a decrease of phosphorylated GATA4 in ob/ob-Erk1 $1^{-/}$compared to $o b / o b$ and $o b /+$ insulin-sensitive control mice (Fig. 3F). miR-144 was also consistently decreased in the livers of $o b / o b$ $\mathrm{Erkl}^{-/-}$mice and in $o b /+$ insulin-sensitive mice (Fig. 3G), leading to increased expression of NRF2 and its target genes (Fig. 3, H and I). These data strongly suggested that miR-144 expression is controlled by GATA4, which is activated by ERK in insulin-resistant mice and humans.

\section{Silencing miR-144 in LMs reduces ROS release and leads to decreased expression of miR-144 in hepatocytes} To investigate the role of $m i R-144$ in the regulation of NRF2 in vivo, we used glucan-encapsulated RNA interference particle $(\mathrm{GeRP})$ technology $(20,21)$ to deliver gene-silencing small interfering RNA specifically in LMs without affecting gene expression in other cells of the liver or the rest of the body. Mice were fed an HFD for 7 weeks and then treated with GeRPs containing an antagomiR targeting $m i R-144$ (amiR-144) or a nontargeting scrambled control (Fig. 4A). We observed a significant knockdown of miR-144 as quantified by qPCR in both LMs and hepatocytes isolated from the GeRP-amiR144-treated mice $(P<0.05$; Fig. $4, \mathrm{~B}$ and $\mathrm{C})$. To assess the specificity of GeRP-mediated silencing of miR-144, we measured the expression of another miRNA, miR-532, which remained unchanged upon treatment with GeRP-amiR-144 (Fig. 4D). 
A

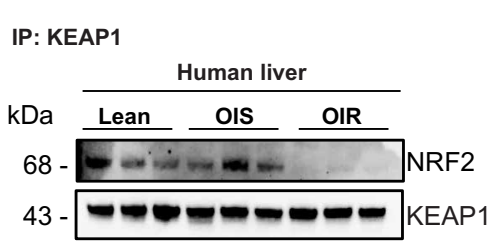

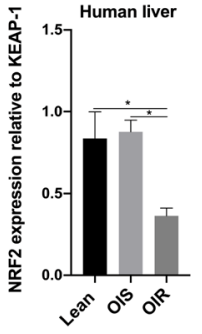

C

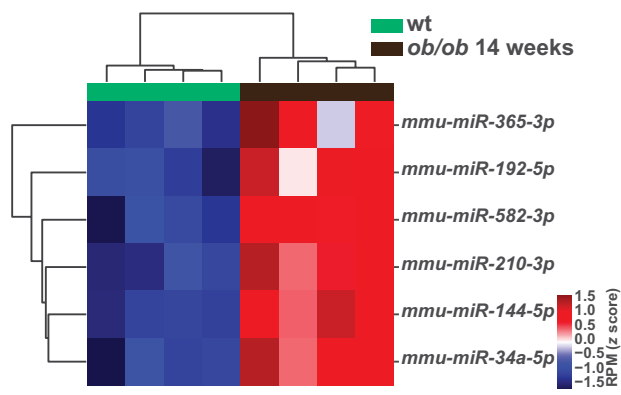

$\mathbf{F}$

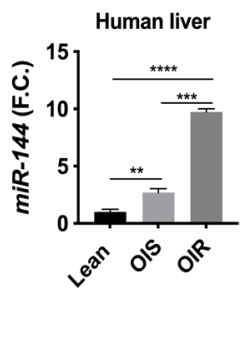

Fig. 2. NRF2 is a target of $\boldsymbol{m i R - 1 4 4}$ in obesity-associated LMs. (A) Western blot of NRF2 and KEAP1 after immunoprecipitation of KEAP1 in the livers from OIS, OIR, and lean human individuals ( $n=3$ per condition). (B and C) Heat maps of significantly (top to down, $P=0.0064, P=0.0013, P=0.0032, P=5.26 \times 10^{-6}, P=0.0029$, and $P=0.0017$ ) up-regulated miRNAs in LMs of 9- (B) and 14- (C) week-old ob/ob compared to wt mice ( $n=4 \mathrm{wt}, n=3$ ob/ob for 9 weeks; $n=4$ per condition for 14 weeks). (D to F) Stem-loop RT-qPCR analysis of miR-144 in LMs (D) and the livers (E) from mice fed an HFD or ND for 9- and 14-week-old $o b / o b$ mice ( $n=3$ per condition) and in the (F) livers from lean, OIS, and OIR human individuals ( $n=5$ per condition). Data are means \pm SEM. RT-qPCR data are F.C. compared to ND mice or lean individuals. ${ }^{*} P<0.05,{ }^{*} P<0.01,{ }^{* *} P<0.001$, and ${ }^{* * *} P<0.0001$. RPM, reads per million.

We next addressed whether the silencing of $m i R-144$ observed in hepatocytes after treatment with GeRP-amiR-144 was specific to this particular miRNA or was a general mechanism affecting all miRNAs. We thus treated mice with GeRPs loaded with an antagomiR targeting another miRNA, miR-192. Treatment with GeRPamiR-192 significantly decreased $m i R-192$ expression in LMs but had no effect on hepatocytes $(P<0.05$; fig. S3, A and B). Furthermore, GeRP-mediated delivery of a miR-192 mimic increased $m i R$ 192 expression in LMs but not hepatocytes isolated from the treated mice (fig. S3, C and D). As GeRP-mediated silencing of miR-144 in hepatocytes seemed specific to this miRNA, we hypothesized that miR-144 could be delivered from LMs to hepatocytes through extracellular vesicles (EVs). To test whether EV delivery could potentially explain why silencing $m i R-144$ in LMs led to decreased expression of miR-144 in hepatocytes, we measured miR-144 abundance in EVs isolated from the media of LMs isolated from mice and treated with antagomiR-144. Although control miRNAs miR-126-3p and UNISP6 were present in this media, $m i R-144$ was undetectable (fig. S3, E to I). An alternative explanation for the decreased expression of $m i R-144$ in hepatocytes after silencing in LMs was a reduction of extracellular ROS, which would no longer induce the transcription of miR-144 in hepatocytes. Thus, we measured the $\mathrm{H}_{2} \mathrm{O}_{2}$ secreted in the media of murine LMs and hepatocytes after silencing miR-144 in LMs. Secretion of $\mathrm{H}_{2} \mathrm{O}_{2}$ was significantly reduced in both LMs and hepatocytes $(P<0.01$; Fig. 4, E and F), suggesting that silencing of miR-144 in LMs could alleviate oxidative stress in the liver microenvironment. We then measured the expression and phosphorylation of GATA4 in hepatocytes after the silencing of $m i R-144$ in LMs isolated from mice. GATA4 phosphorylation was reduced in hepatocytes upon treatment with GeRP-amiR-144 (Fig. 4G), corroborating the notion that silencing miR-144 in LMs isolated from mice leads to a reduction in miR-144 transcription in hepatocytes.

To further investigate the regulation of $m i R-144$ and ROS secretion, we exposed human LMs to different concentrations of $\mathrm{H}_{2} \mathrm{O}_{2}$ and silenced $m i R-144$ using antagomir amiR-144 in vitro. $\mathrm{H}_{2} \mathrm{O}_{2}$ treatment increased expression of GATA4 leading to increased miR-144 expression (fig. S3, J and K) that was blunted by amiR-144 (Fig. $4 \mathrm{H}$ ). As expected, $\mathrm{H}_{2} \mathrm{O}_{2}$ significantly increased the expression of NRF2 antioxidant target gene NQO1 $(P<0.001)$, which was further enhanced by silencing miR-144 $(P<$ 0.0001; fig. S3L). After $\mathrm{H}_{2} \mathrm{O}_{2}$ treatment, human LMs secreted significantly more $\mathrm{H}_{2} \mathrm{O}_{2}(P<0.0001)$, which was mitigated by amiR-144 $(P<0.0001$; Fig. 4I), suggesting a feedback loop between extracellular ROS, intracellular ROS, and miR-144/GATA4 expression.

Using a three-dimensional (3D) culture model of human primary hepatocytes (liver spheroids) (22), we found that treatment with extracellular $\mathrm{H}_{2} \mathrm{O}_{2}$ was sufficient to induce expression of miR-144 and NRF2 target gene NQO1 (fig. S3, M and N). To more closely mimic the in vivo liver environment, we added nonparenchymal cells (NPCs) and with free fatty acids (FFAs) to the spheroids to recapitulate the lipid overload in obese livers. Four types of liver spheroids were formed: those with normal miR-144 expression in both hepatocytes and NPCs, those with miR-144 silenced only in NPCs or only in hepatocytes, and those with $m i R-144$ silenced in both populations (Fig. 4J). As expected, treatment with FFAs boosted miR-144 expression in liver spheroids, whereas silencing of $m i R-144$ in the 
Table 1. Parameters of study individuals. Code, patient-specific identifier. BMI, body mass index. HOMA-IR, homeostatic model assessment of insulin resistance. HIS, hepatic steatosis index. Insulin sensitivity assessed by HOMA-IR method.

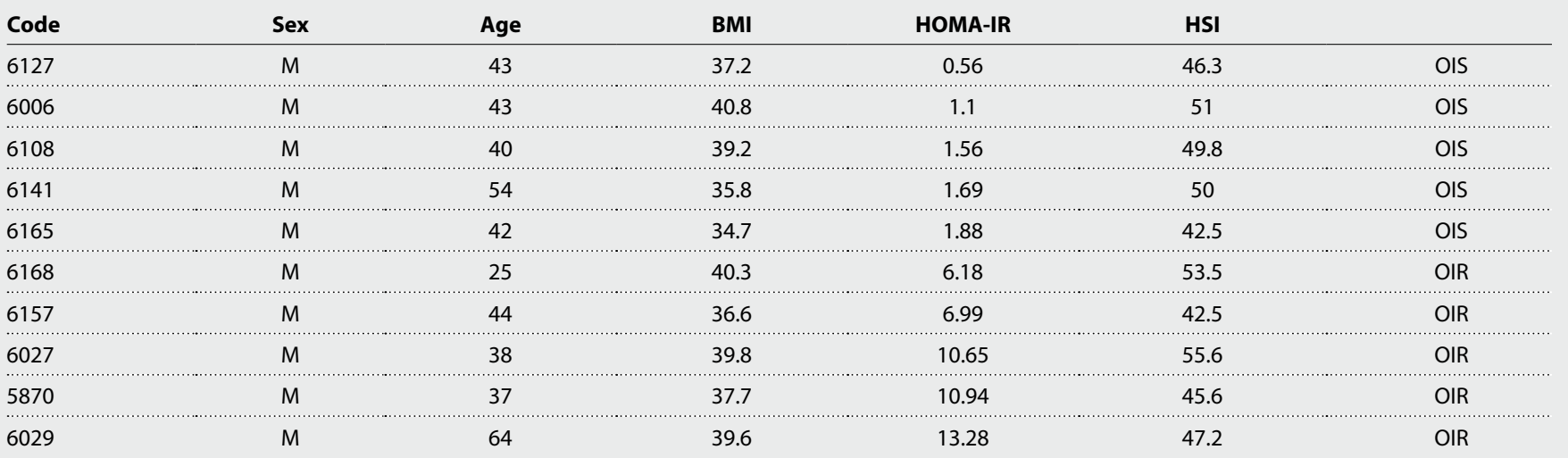

NPCs alone reduced FFA-driven induction of miR-144 (Fig. 4K), as observed in the mouse model. Moreover, antagomiR-144 administration in hepatocytes alone or in hepatocytes and NPCs together decreased miR-144 in spheroids treated with FFAs (Fig. 4K). miR-144 silencing increased NRF2 (Fig. 4L) and the expression of NRF2 antioxidant target gene NQO1 (Fig. 4M), leading to a significant decrease in the generation of ROS induced by FFA $(P<0.001$; Fig. $4 \mathrm{~N})$. These results highlight the importance of $m i R-144$ expressed by LMs and hepatocytes in the regulation of the endogenous antioxidant response. Considering the low percentage of LMs in the liver (6 to 10\%) (10), these results support the importance of LMs in the regulation of $m i R-144$ expression and ROS secretion in the liver during obesity in mice and humans.

\section{Silencing miR-144 in LMs reduces oxidative stress and improves hepatic metabolism in insulin resistance in mice}

Silencing $m i R-144$ specifically in LMs reduced the expression of miR-144 in hepatocytes via reduction of GATA4 phosphorylation. Because GATA4 phosphorylation is triggered by oxidative stress, we hypothesized that $m i R-144$ silencing in LMs could reduce oxidative stress in the obese liver. We first measured NRF2, ROS, and RNS in the livers of obese mice treated with either GeRP-amiR-144 or a GeRP scrambled control (GeRP-scr). We observed a significant increase of NRF2 in the livers of mice treated with GeRP-amiR-144 in whole livers, LMs, and hepatocytes $(P<0.001, P<0.001$, and $P<0.05$, respectively; Fig. 5, A to C), along with increased expression of $N r f 2$ target genes (Nqo1, Gstp1, and Ces2G) (Fig. 5, D and E). Silencing miR-144 in LMs reduced ROS and, to a lesser extent, RNS in the livers of treated mice compared to controls (Fig. 5, F to H). This result confirmed the hypothesis that silencing miR-144 in LMs resulted in reduction of miR-144 expression in hepatocytes due to a decreased production of both ROS and RNS. To further investigate this mechanism, we used amiR-144 to silence $m i R-144$ in lean healthy mice that produced physiological concentrations of ROS and RNS. After treatment with fluorescein isothiocyanate (FITC)-labeled GeRPs, LMs containing GeRPs $\left(\mathrm{CD} 45^{+} / \mathrm{F} 4 / 80^{+} / \mathrm{Cd}_{1} 1 \mathrm{~b}^{+} / \mathrm{FITC}^{+}\right)$, empty LMs $\left(\mathrm{CD}^{+} 5^{+} /\right.$ $\left.\mathrm{F} 4 / 80^{+} / \mathrm{Cd} 11 \mathrm{~b}^{+} / \mathrm{FITC}^{-}\right)$, and empty non-LM NPCs $\left(\mathrm{CD} 45^{-} / \mathrm{FITC}^{-}\right)$ were sorted by flow cytometry, and hepatocytes were isolated. amiR-144 treatment did not influence the percentage of resident or recruited macrophages (Fig. 5I). Moreover, although miR-144 was successfully silenced in FITC ${ }^{+}$LMs, we observed no effect on the expression of $m i R-144$ in any other cell fractions (Fig. 5, J to M). These data further confirmed that the reduction in miR-144 in hepatocytes after silencing in LMs was due to a decreased oxidative stress leading to diminished transcription of miR-144 via GATA4.

We then assessed whether the increase in NRF2 and reduced liver oxidative stress had an effect on whole-body metabolism. We did not observe changes in body weight or total triglyceride content in the livers upon treatment with GeRP-amiR-144 (fig. S4, A to C). However, transmission electronic microscopy (TEM) revealed an increase in the number of mitochondria after miR-144 silencing, suggesting an adaptive mechanism to protect hepatocytes against oxidative stress (Fig. 5, N and O). Stored intracellular glycogen in the liver was increased in mice treated with GeRP-amiR-144 (Fig. 5P). We thus assessed whether silencing miR-144 could affect wholebody glucose metabolism. Consistent with the increased glycogen stores, glucose tolerance tests showed improved glucose homeostasis in mice treated with GeRP-amiR-144 compared to control mice (Fig. 5Q). This effect was specific for miR-144 because we did not detect any differences after treatment with GeRP-amiR-192 (fig. S4D). These data suggested that miR-144 expressed by LMs and hepatocytes contribute to liver oxidative stress and glucose homeostasis in obesity. Together, these results demonstrate that $m i R-144$ decreased NRF2 protein in the liver, resulting in an impaired antioxidant response in obese insulin-resistant mice and humans (fig. S5).

\section{DISCUSSION}

In this study, we investigated the role of LMs in the regulation of the antioxidant response in the livers of obese insulin-resistant humans and mice. Previous studies suggested that oxidative stress and associated damage could represent a link between obesity and liver disease (23-26). We confirmed that oxidative stress was triggered by obesity in murine and human livers and showed that lipid oxidation and the antioxidative response were among the most impaired pathways in two models of obesity.

The main mechanism protecting against oxidative stress in mice and in humans is the NRF2/ARE pathway, which induces the expression of antioxidant response genes (27) and directly regulates 
A

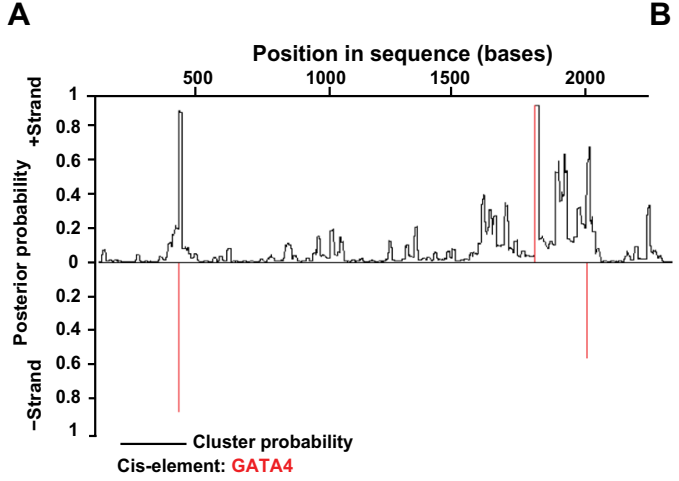

C

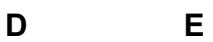

B

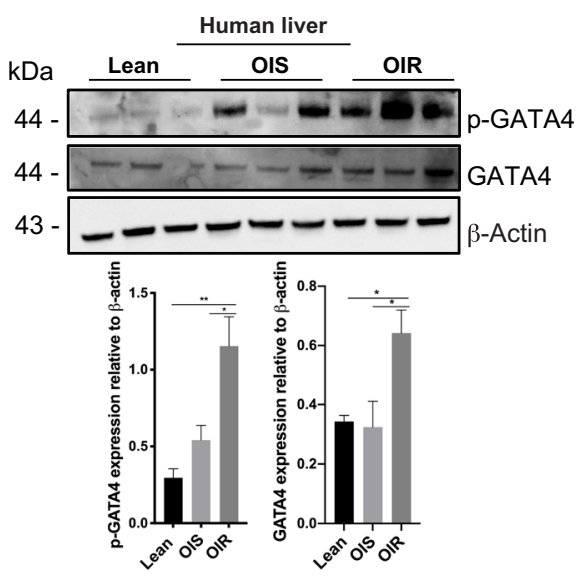

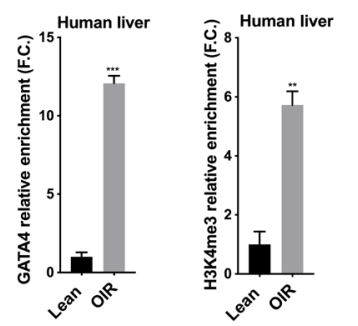

$\mathbf{F}$

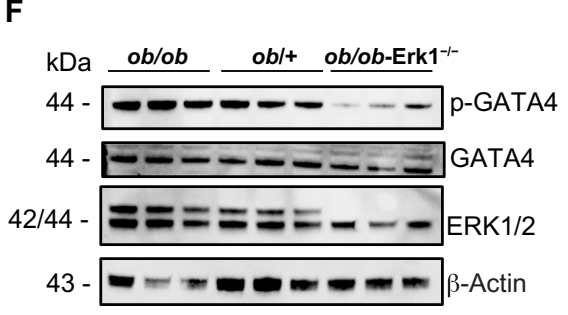

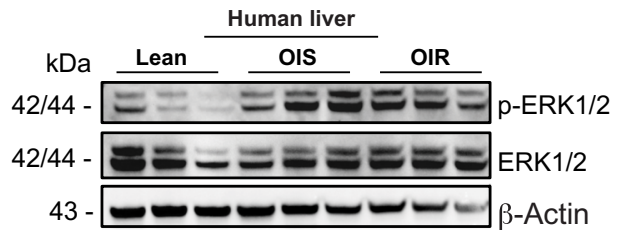

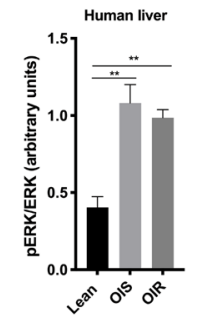

H

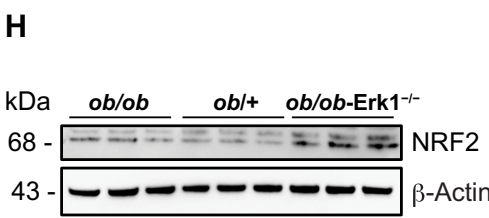

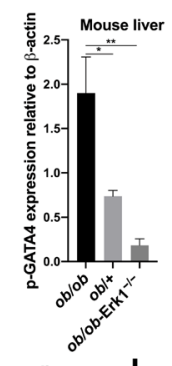

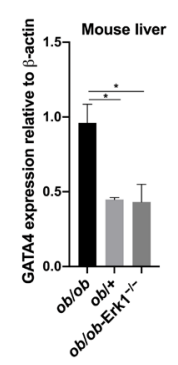

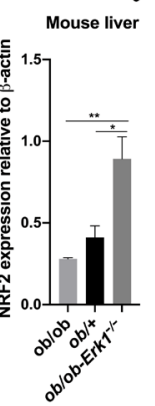

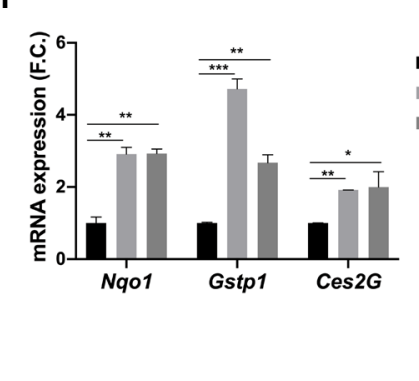

Fig. 3. The transcription factor GATA4 drives the expression of $m i r-144$ in the liver of insulin-resistant individuals. (A) In silico prediction of GATA4-binding domains on the miR-144 promoter region. (B) Western blots showing phosphorylated GATA4 ( $p$-GATA4) and GATA4 in the livers from lean, OIS, and OIR human individuals ( $n=3$ per condition). (C and D) ChIP-qPCR of GATA4 and H3K4me3 relative enrichment on the miR-144 promoter in the livers from lean and OIR human individuals ( $n=3$ per condition). Data are normalized by immunoglobulin $\mathrm{G}$ and F.C. compared to lean participants. (E) Western blots showing phosphorylated ERK1/2 (p-ERK1/2) and ERK1/2 in the livers from lean, OIS, and OIR human individuals ( $n=3$ per condition). Quantification of $\mathrm{p}$-ERK1/2/ERK $1 / 2$ ratio. (F) Western blots of $\mathrm{p}$-GATA4, GATA4, and ERK1/2 in the livers from $o b /+, o b / o b$, and $o b / o b-E r k 7^{-1-}$ mice $(n=7$ per condition). (G) Stem-loop RT-qPCR analysis of miR-144 in the livers from ob/+,ob/ob, and ob/ob-Erk $1^{-/-}$mice ( $n=7$ per condition). (H) NRF2 in the livers from $o b /+, o b / o b$, and $o b / o b-E r k 1^{-/-}$mice ( $n=3$ per condition). (I) RT-qPCR analysis of Nrf2 target genes Nqo1, Gstp1, and Ces2G in the livers from ob/+,ob/ob, and ob/ob-Erk $1^{-/-}$mice ( $n=3$ per condition). RT-qPCR data are F.C. compared to $o b /+$. Data are means \pm SEM. ${ }^{*} P<0.05,{ }^{* *} P<0.01$, and ${ }^{* *} P<0.001$.

the homeostasis of both ROS and RNS (28). We found that NRF2 protein expression was reduced in obese mice and human individuals, suggesting an impaired antioxidant response. KEAP1 has been ex-

G

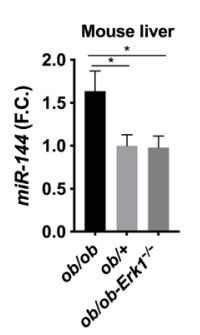

tensively described as the main regulator of NRF2 at the posttranscriptional level. In the absence of oxidative stress, the interaction between NRF2 with KEAP1 facilitates the proteasomal degradation and rapid turnover of NRF2 $(27,28)$. Conversely, under conditions of oxidative stress, the modification of KEAP1 cysteine residues leads to a change in its conformation that releases NRF2, which then translocates to the nucleus where it binds to the ARE, subsequently activating the transcription of antioxidant genes $(29,30)$. Inflammatory activation of macrophages has been associated with a higher production of itaconate from citrate in the TCA cycle, which then activates NRF2 through alkylation of KEAP1 (31). In that context, itaconate was described as an anti-inflammatory metabolite able to reduce oxidative stress.

In this study, we found that $N r f 2$ mRNA expression remained unchanged upon oxidative stress induced by obesity. Furthermore, neither expression of KEAP1 nor ubiquitination of NRF2 changed during obesity, suggesting a posttranscriptional mechanism regulating NRF2 protein independently of its interaction with KEAP1. Considering that LMs do not undergo inflammatory activation during obesity, the different NRF2 regulation could depend on the type of stimulus and its kinetics. A previous study described a KEAP1-dependent regulation of NRF2 upon inflammatory activation of macrophages by a potent and acute inflammatory stimulus (lipopolysaccharide or interferon- $\beta$ ) (31). However, macrophages in our study were exposed to chronic lipid overload resulting in oxidative stress that did not induce inflammatory activation and may require a more sustainable mechanism of regulation (for example, via miR-144) than rapid degradation (such as via KEAP1). We also do not exclude the possibility of a differential mechanism of regulation due to the use of different macrophage cell types (blood- and bone marrow-derived macrophages versus LMs).

We hypothesized that NRF2 protein could be targeted by an miRNA, and thus we analyzed the miRNome of LMs from obese and healthy mice. Among the up-regulated miRNAs we detected in obese LMs, miR-144 has previously been reported to reduce NRF2 protein levels in cancer (32). We found that miR-144 expression was highly increased in whole livers of obese mice and humans, and 


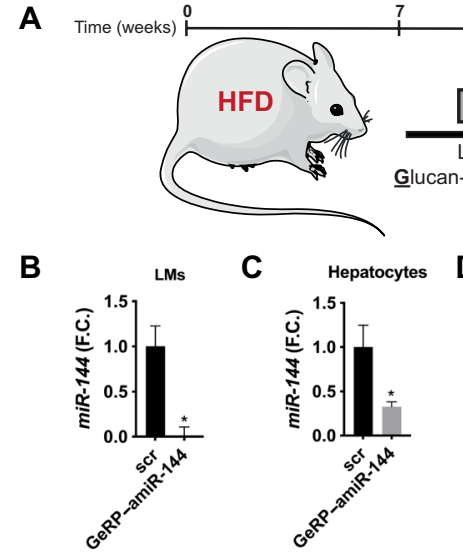

G

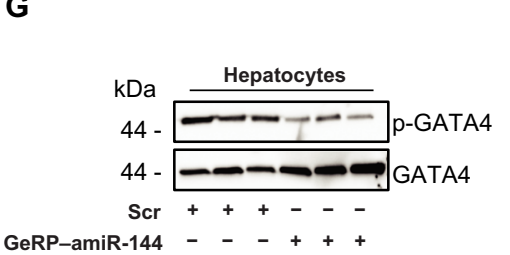

GeRP-amiR-144 - - + + +

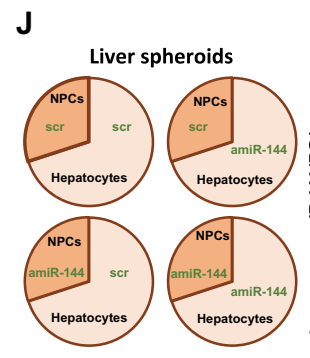

$\mathbf{L}$
K
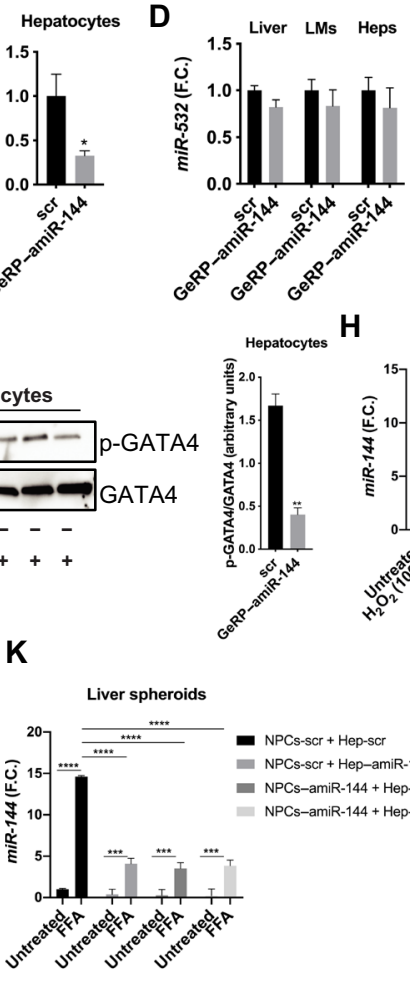

$\mathbf{F}$

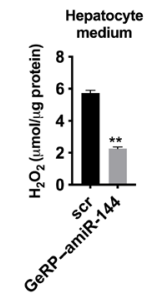

E

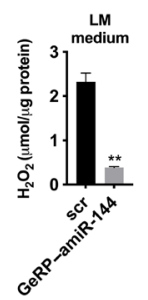

- Effect on glucose tolerance - Cell and tissue collection
H Human LMs I

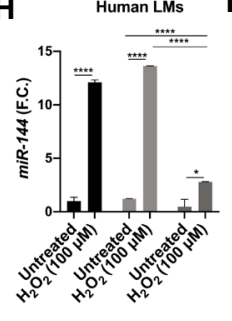

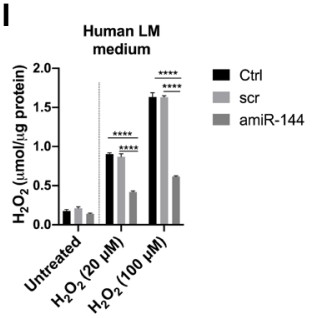

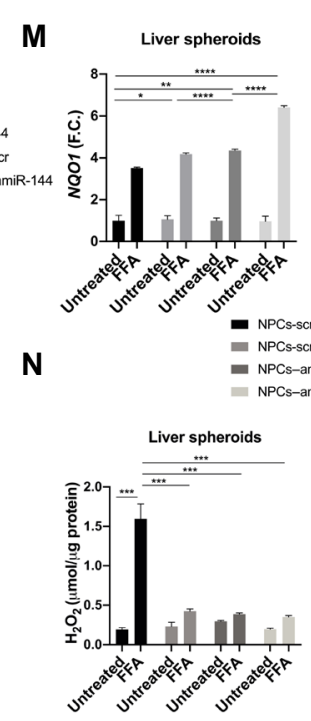

Fig. 4. Silencing miR-144 in LMs reduces ROS release and leads to decreased expression of $m i R-144$ in hepatocytes. (A) Protocol of GeRP-amiR-144 treatment. (B and C) Stem-loop RT-qPCR analysis of miR-144 in LMs (B) and hepatocytes (C) from GeRP-scrambled control (scr)- and GeRP-amiR-144-treated mice ( $n=4$ per condition). (D) Stem-loop RT-qPCR analysis of miR-532 in livers, LMs, and hepatocytes from GeRP-amiR-144- and scr-treated mice ( $n=4$ per condition). (E and $\mathbf{F})$ Extracellular $\mathrm{H}_{2} \mathrm{O}_{2}$ content in media from mice $L M s(E)$ and hepatocytes ( $F$ ) treated with scr or GeRP-amiR-144 ( $n=4$ per condition). (G) Western blot analysis of p-GATA4 and GATA4 in hepatocytes from scr-and GeRP-amiR-144-treated mice ( $n=3$ per condition). Quantification of p-GATA4/GATA4 ratio. (H) Stem-loop RT-qPCR analysis of miR-144 in human LMs untreated, exposed $100 \mu \mathrm{M} \mathrm{H}_{2} \mathrm{O}_{2}$ and treated with scr or GeRP-amiR-144 ( $n=3$ per condition). (I) Extracellular $\mathrm{H}_{2} \mathrm{O}_{2}$ content in media from human $\mathrm{LMs}$ untreated or exposed to $100 \mu \mathrm{M} \mathrm{H}_{2} \mathrm{O}_{2}$ and treated with scr or GeRP-amiR-144 ( $n=3$ per condition). ( $(J)$ Schematic representation of the proportion of liver spheroid cell types and treatments. (K) Stem-loop RT-qPCR of miR-144 in hepatocytes and NPCs from human liver spheroids exposed to FFA and treated with amiR-144 or scr (pooled liver spheroids from three human donors). (L) NRF2 in human liver spheroids exposed to FFA and treated with amiR-144 or scr (pooled liver spheroids from three human donors). (M) RT-qPCR analysis of NRF2 target gene NQO1 in human liver spheroids exposed to FFA and treated with amiR-144 or scr (pooled liver spheroids from three human donors). (N) Extracellular $\mathrm{H}_{2} \mathrm{O}_{2}$ content in media from human liver spheroids exposed to FFA and treated with amiR-144 or scr (pooled liver spheroids from three human donors). Data are means \pm SEM. RT-qPCR data are F.C. compared to scr. ${ }^{*} P<0.05,{ }^{* *} P<0.01,{ }^{* * *} P<0.001$, and $* * * * P<0.0001$.

insulin resistance was associated with an increase in $m i R-144$ in humans. To study the mechanism whereby $m i R-144$ was regulated by insulin resistance, we performed an in silico predictive analysis, which detected binding sites for the transcription factor GATA4 near the miR-144 transcriptional start site. ChIP analysis revealed that GATA4 indeed bound the promoter region of $m i R-144$ to induce its transcription. This is consistent with reports that miR-144 transcription is regulated by the transcription factor GATA4 in cardiomyocytes (33). In mice, GATA4 activation via ERKmediated phosphorylation in cardiomyocytes has been previously shown to be induced by hyperglycemia (18). Our investigations corroborated these findings because we observed increased ERK phosphorylation in obese patients compared to lean controls. In addition, GATA4 phosphorylation was reduced in the livers of obese $\mathrm{Erk1}^{-/-}$mice, and consequently, miR-144 and NRF2 remained unchanged upon obesity. We note that whereas miR-144 was increased in insulin-resistant compared to insulin-sensitive obese participants, ERK1/2 phosphorylation was comparable between the two groups. However, GATA4 protein expression was higher in obese insulin-resistant individuals, suggesting that the increased expression of $m i R-144$ in these individuals might not only be due to the activation of GATA4 but also to its increased abundance.

Taking advantage of GeRP technology to specifically manipulate gene expression in LMs, we observed a decrease in miR-144 in LMs and hepatocytes. This result was unexpected because GeRPs cannot be delivered to nonphagocytic cells such as hepatocytes $(10,34,35)$. We confirmed the specific liver cell distribution of GeRPs by targeting another miRNA, miR-192, which was only silenced in LMs and not in hepatocytes. On the basis of these findings, we hypothesized that LMs could deliver miR-144 to hepatocytes through EVs, and silencing miR-144 in LMs could therefore result in decreased miR-144 in both LMs and hepatocytes. However, EVs produced by LMs did not contain miR-144, which remained undetectable after silencing of $m i R-144$.

The other possible explanation for the concurrent GeRP-driven down-regulation 
A

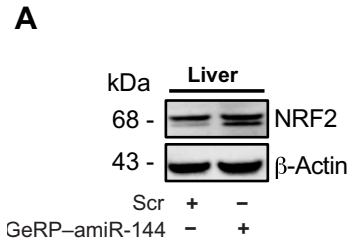

C

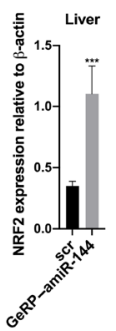

B
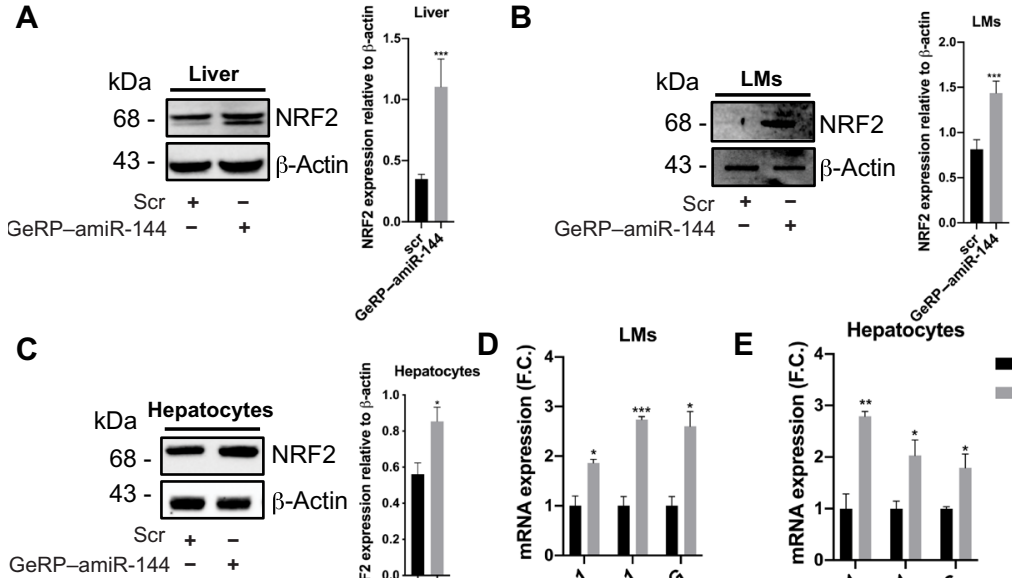

GeRP-amiR-144 - +
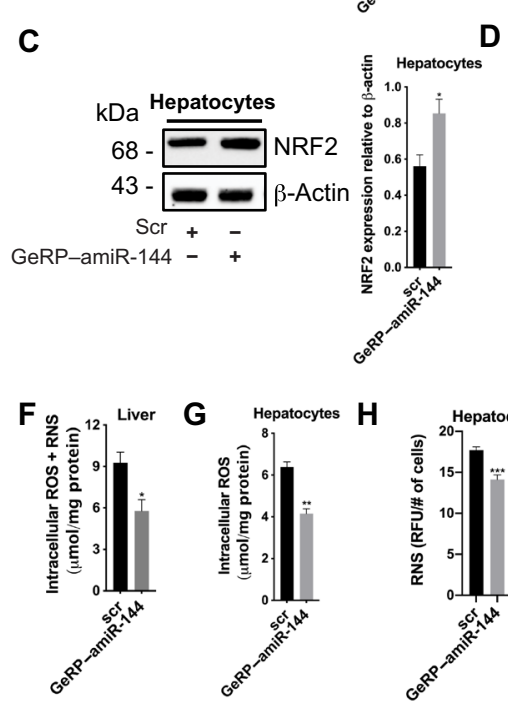

G Hepatocytes $\mathbf{H}$

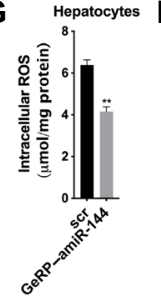

K

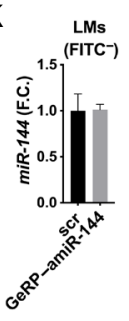

$\mathbf{L}$
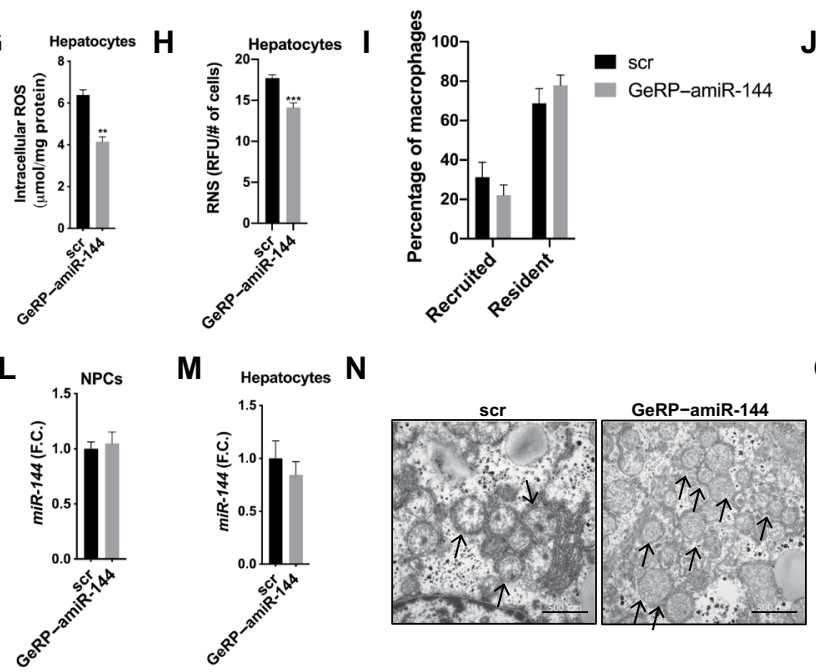

M Hepatocytes $\mathbf{N}$
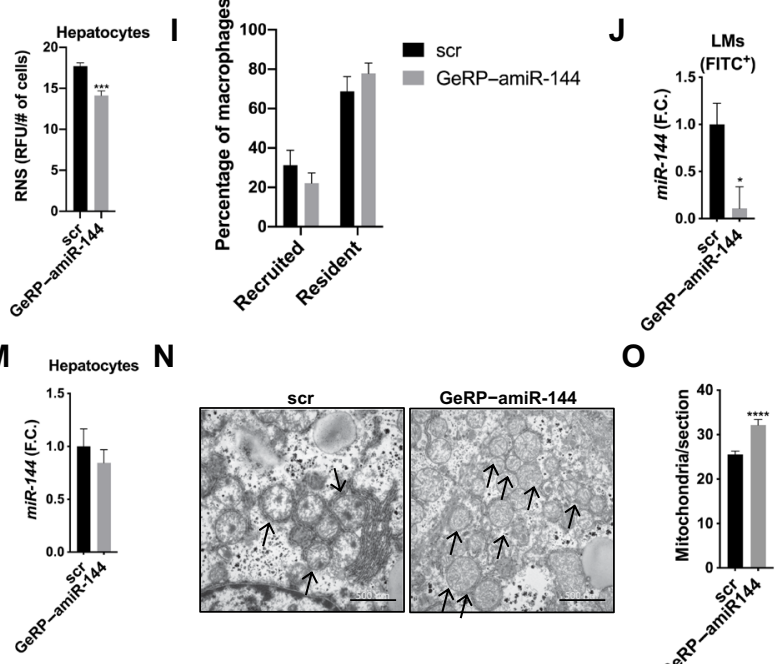

$\mathbf{P}$

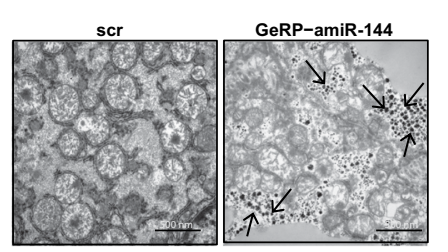

Q

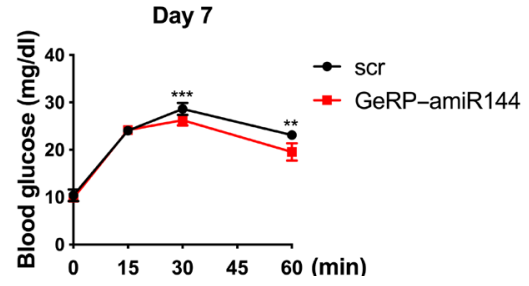

Fig. 5. Silencing miR-144 in LMs reduces oxidative stress and improves hepatic metabolism in insulin resistance. (A to C) NRF2 in mouse livers (A), LMs (B), and hepatocytes (C) treated with scrambled control (scr) or GeRP-amiR-144 ( $n=4$ per condition). (D and E) RT-qPCR analysis of NRF2 target genes Nqo1, Gstp1, and Ces2G in LMs and hepatocytes from scr- and GeRP-amiR-144-treated mice ( $n=4$ per condition). (F) Total intracellular ROS/RNS content in the livers from scr- and GeRP-amiR-144-treated mice ( $n=4$ per condition). ( $\mathbf{G}$ and $\mathbf{H}$ ) Intracellular ROS (G) and RNS (H) in hepatocytes from scr-and GeRP-amiR-144-treated mice ( $n=4$ per condition). (I) Percentage of resident and recruited macrophages from scr- and GeRP-amiR-144-treated ND-fed mice ( $n=4$ per condition). (J to $\mathbf{L}$ ) Stem-loop RT-qPCR analysis of miR-144 in (J) CD45 $/$ F4 $/ 80^{+} / \mathrm{Cd}_{11} 1 \mathrm{~b}^{+} / \mathrm{FITC}^{+} \mathrm{LMs}$, (K) CD45 $/ \mathrm{F} 4 / 80^{+} / \mathrm{Cd} 11 \mathrm{~b}^{+} / \mathrm{FITC}^{-} \mathrm{LMs}$, and (L) CD $45^{-} / \mathrm{FITC}^{-}$ nonparenchymal cells (NPCs) from scr-and GeRP-amiR-144-treated ND-fed mice ( $n=4$ per condition). (M) Stem-loop RT-qPCR analysis of miR-144 in hepatocytes from scr- and GeRP-amiR-144-treated ND-fed mice ( $n=4$ per condition). (N) TEM showing an increased number of mitochondria in the livers from scr-and GeRP-amiR-144-treated mice $(n=2$ per condition). Black arrows depict mitochondria. (0) Number of mitochondria per section in the livers from scr-and GeRP-amiR-144-treated mice ( $n=20$ sections per condition). (P) TEM showing increased stored glycogen in the livers from scr- and GeRP-amiR-144-treated mice ( $n=2$ per condition). Black arrows depict glycogen deposits. (Q) IP-GTT of scr- and GeRP-amiR-144-treated mice ( $n=5$ per condition). Data are means \pm SEM. RT-qPCRs data are F.C. compared to scr. ${ }^{*} P<0.05,{ }^{* *} P<0.01,{ }^{* * *} P<0.001$, and ${ }^{* * *} P<0.0001$.

of miR-144 in both LMs and hepatocytes was that silencing $m i R-144$ in LMs may have reduced ROS and RNS production in the liver and consequently decreased the expression of $m i R-144$ in hepatocytes. Consistent with this hypothesis, knockdown of miR-144 in LMs diminished oxidative stress markers in whole livers of obese mice, suggesting cross-talk between LMs and hepatocytes. To test this hypothesis, we focused on $\mathrm{H}_{2} \mathrm{O}_{2}$ extracellular release from LMs because $\mathrm{H}_{2} \mathrm{O}_{2}$ is considered one of the most stable ROS and can cross membranes unlike other ROS $(36,37)$. Our findings revealed that ROS release by both LMs and hepatocytes decreased after miR-144 silencing in LMs. However, we found that hepatocytes may also play a role in the regulation of miR-144. Silencing miR-144 in human primary hepatocyte 3D cultures (22) exposed to $\mathrm{H}_{2} \mathrm{O}_{2}$ or FFAs was able to efficiently trigger the antioxidant response. ROS and possibly RNS could therefore potentially act as secondary messengers that contribute to a vicious cycle, whereby LMs communicate with hepatocytes to increase $m i R-144$ expression, leading to an impaired antioxidant response. ROS release has been mainly described in proinflammatory macrophages $(28,38-40)$, whereas we found that ROS production could be dissociated from inflammation in LMs in obesity. In addition, GATA4 phosphorylation was reduced in hepatocytes upon silencing of miR-144 in LMs, confirming a major role of LMs in the regulation of $m i R-144$ transcription induced by oxidative stress. Because NRF2 protein was increased upon $m i R-144$ silencing in LMs, the observed reduction in oxidative stress in these cells could be explained by a restored endogenous antioxidant response. Although additional work will be needed to study the mechanism whereby NRF2 restoration drives the antioxidant response at the subcellular level, the increased number of mitochondria in the livers of mice treated with the antagomiR-144 suggests an effect on mitochondrial biogenesis, as previously described $(41,42)$.

Last, silencing $m i R-144$ expression in LMs improved glucose tolerance and increased liver glycogen stores in obese mice. Consistent with a role of ERK1/2 in the activation of GATA4 and subsequent increase in $m i R-144, o b / o b-E r k 1^{-/}$, and 
obese mice treated with GeRP-amiR-144 shared a similar phenotype (19). Although the molecular mechanism whereby Erk 1 deficiency improved metabolism in $o b / o b$ mice is not fully elucidated, our findings suggest that a better antioxidant response might explain the improved liver function in these mice. We demonstrated that silencing miR-144 in human LMs previously treated with $\mathrm{H}_{2} \mathrm{O}_{2}$ induced the endogenous antioxidant response and reduced GATA4 expression, suggesting a feedback loop between GATA4 and miR-144 transcriptions in response to ROS.

Although several studies have highlighted the beneficial effects of NRF2 activation $(43,44)$, long-term NRF2 stimulation has been associated with liver fibrosis (45), reductive stress (46), and promotion of preexisting malignancies (47). The major advantage of GeRP technology is its ability to manipulate miRNA expression in a transient and specific manner in LMs while leaving other cells and macrophages in the body unaffected. This is particularly important because in vivo attempts to reduce oxidative stress using exogenous antioxidants such as vitamin $\mathrm{C}$, vitamin $\mathrm{E}$, or $\beta$-carotene have had no beneficial or even deleterious effects (48-50). The lack of efficacy of these exogenous antioxidants is thought to be due to both nonspecific systemic effects and a decrease in the endogenous antioxidant response, highlighting the importance of a targeted approach to increase the endogenous antioxidant response to reduce oxidative stress.

In sum, this study unveils the important role of LMs in the regulation of systemic metabolism in obesity-induced insulin resistance in mice and humans. Specifically, LMs produce miRNAs that impair the antioxidant capacity of the liver in response to excessive lipid accumulation as observed in obesity-associated insulin resistance. We cannot exclude the importance of hepatocytes in the regulation of miR-144 expression and of the antioxidant response in obesity. However, despite the low percentage of LMs in the liver, we observed a global antioxidant effect of $m i R-144-$ specific silencing in LMs, highlighting the importance of LMs in the regulation of the endogenous antioxidant response in obesity.

There are limitations to our work. Although silencing $m i R-144$ led to a systemic effect on glucose metabolism, we did not observe any change in hepatic steatosis and liver triglyceride content. However, our data demonstrated that silencing of $m i R-144$ affected the cellular response to oxidative stress induced by excess lipid accumulation in the liver, rather than lipid deposition itself. Thus, although we do not think that a longer treatment would have an effect on hepatic steatosis, it would be interesting to silence $m i R-144$ over a longer treatment period than 15 days to address this matter.

Moreover, although our study highlighted the critical cross-talk between LMs and hepatocytes in the up-regulation of oxidative stress during obesity, we understand that a better characterization of the specific ROS and RNS populations involved in this process is needed. Although ROS and RNS share analogies in their production, function, and decomposition (51), our results focused on $\mathrm{H}_{2} \mathrm{O}_{2}$ release, one of the most stable ROS extracellularly $(36,37)$. Because NRF2 regulates both populations (28), we cannot exclude contribution(s) of other ROS or RNS.

Although our study clearly shows that the antioxidant response is impaired in obese insulin-resistant patients and animals through overexpression of $m i R-144$ targeting NRF2, further work will be required to validate the role of $m i R-144$ at different points of liver diseases. NAFLD encompasses a spectrum of disease stages from steatosis to nonalcoholic steatohepatitis (NASH) and eventually fibrosis leading to cirrhosis and hepatocarcinoma (HCC). We focused our investigation on the initial step (insulin resistance and steatosis). However, it would be important to analyze the roles of $m i R-144$, NRF2, and oxidative stress at these various phases of fatty liver diseases to understand the role of $m i R-144$ in the development of NASH and HCC. Last, although we demonstrated that $m i R-144$ plays an important role in the regulation of the antioxidant response, our experiments were performed using bulk analyses that do not provide information at the single-cell level. Additional single cell-based assays such as single-cell RNA-seq will be required to clearly define whether macrophage subpopulations are predominantly affected by insulin resistance and oxidative stress. Despite these limitations, our study suggests that specific targeting of LMs to attenuate the burden of oxidative stress during obesity could represent a therapeutic approach for metabolic diseases, which are often associated with chronic liver diseases.

\section{MATERIALS AND METHODS Study design}

The objective of this study was to investigate the contribution of LMs to the oxidative stress associated with obesity-induced insulin resistance in mice and humans. To confirm oxidative stress in the livers of obese mice and humans, we measured the content of ROS/RNS, lipid content, and malonyl-dehaldehyde. We measured the protein expression of its master regulator, NRF2, to assess the endogenous antioxidant response. To analyze the molecular mechanisms involved in NRF2 posttranscriptional regulation, we performed small RNA-seq on LMs of lean and obese mice and qPCR in the livers of mice and humans. We demonstrated that $m i R-144$ targets NRF2 in liver tissues obtained from various mouse and human cohorts. Power analyses were not used to calculate sample sizes; samples were not excluded, and investigators were not blinded during experiments. Before treatment, mice were weighed, and glucose tolerance tests were performed. Mice were then assigned to treatment groups to ensure similar average initial body weights and glucose intolerance. About three to five mice were included in each treatment group as indicated in figure legends, and body weights were continuously monitored weekly throughout treatment with GeRPs loaded with control scrambled or amiR-144. Lean, obese insulin-sensitive, and obese insulin-resistant human individuals were scored according to the homeostatic model assessment of insulin resistance (HOMA-IR). Obese patients were matched for body mass index (BMI). We used five human liver biopsies per condition. For in vitro metabolic studies with liver spheroids, three independent experiments were performed. Primary data are reported in data file S2.

\section{Human participants}

We obtained liver samples from a total of 15 individuals, including 10 obese patients (BMI between 35 and $42 \mathrm{~kg} / \mathrm{m}^{2}$ ) undergoing laparoscopic Roux-en-Y gastric bypass surgery at Danderyd hospital or Ersta hospital in Stockholm, Sweden. We obtained liver cells from five nonobese patients and isolated by the Liver Cell Laboratory at the Unit of Transplantation surgery, Department of Clinical Science, Intervention and Technology at Karolinska Institutet, Sweden. None of the participants had any previous history of cardiovascular disease, diabetes, gastrointestinal disease, systemic illness, alcohol abuse, coagulopathy, chronic inflammatory disease, any clinical sign of liver damage, or surgical intervention within 6 months before the study. Patients did not follow any special diet before the surgery. Insulin sensitivity was assessed by homeostatic model assessment (HOMA-IR). 
Of the obese patients, five patients with HOMA-IR $<2$ were defined as obese insulin sensitive and five with HOMA-IR $>4$ as obese insulin resistant. Hepatic steatosis index (HSI) was calculated as in (52). The Regional Ethical Committee in Stockholm approved the study, and all the participants gave written informed consent for all procedures before their participation. Liver cells from nonobese patients were obtained from liver donors and isolated by the Liver Cell Laboratory at the Unit of Transplantation Surgery, Department of Clinical Science, Intervention and Technology at Karolinska Institutet.

\section{Mice and diet}

Four-week-old wt C57BL/6J and five-week-old $o b / o b$ males were obtained from Charles River Laboratories International Inc. and maintained on a 12-hour light/dark cycle. Animals were given free access to food and water. C57BL/6J wt mice were fed an HFD composed of $60 \%$ calories from fat, $20 \%$ from carbohydrates, and $20 \%$ from protein (Research Diets Inc.) at 5 weeks of age. Control mice were fed a normal chow diet. All experiments were performed at 9 weeks for HFD mice, or at 9 and 14 weeks for $o b / o b$ mice. All procedures were performed in accordance with guidelines approved by the Ethical Committee in Stockholm (Stockholms södra djurförsöksetiska nämnd).

\section{GeRP administration by intravenous injection in vivo}

GeRPs were prepared as previously described (15). The wt mice fed an HFD for 8 weeks were randomized to groups according to their body weight and glucose tolerance. Mice were then treated with GeRPs $(12.5 \mathrm{mg} / \mathrm{kg}$ ) loaded with miRIDIAN miRNA mmu-miR144-5p hairpin inhibitor (GeRP-amiR-144) (Dharmacon) or with miRIDIAN miRNA hairpin inhibitor negative control no. 1 (Dharmacon) $(247 \mu \mathrm{g} / \mathrm{kg})$ and Endoporter $(2.27 \mathrm{mg} / \mathrm{kg})$ as a nontargeting scrambled control. Mice received six doses of fluorescently labeled GeRPs by intravenous injection over 15 days.

\section{Metabolic analyses in mice}

Glucose tolerance tests (IP-GTTs) were performed on the day of the last GeRP injection after a 6-hour fast. A dose of glucose $(1 \mathrm{~g} / \mathrm{kg})$ was injected intraperitoneally, and blood glucose concentrations were measured using a glucometer at defined time points from the tail vein. The following day, mice were euthanized, and tissues were collected for subsequent analyses.

\section{Statistical analysis}

Statistical significance of differences among groups was analyzed whenever appropriate with one-way or two-way analysis of variance (ANOVA) with appropriate post hoc analyses using GraphPad Prism 8.0 or nonparametric Mann-Whitney $U$ tests. Data were presented as means \pm SEM. $P$ values $<0.05$ were considered as statistically significant. Sample sizes for each experiment were calculated on the basis of previous data collection and as described in (53). Although we started every in vivo experiment with the same number of animals per group, if any individual animal showed any sign of discomfort or an injection failed, we terminated the study for this particular animal in accordance with our ethical permit.

\section{SUPPLEMENTARY MATERIALS}

stm.sciencemag.org/cgi/content/full/12/532/eaaw9709/DC1 Materials and Methods

Fig. S1. Oxidative stress in LMs fails to trigger an antioxidant response in obesity-induced insulin resistance.
Fig. S2. NRF2 is a target of miR-144 in obesity-associated LMs.

Fig. S3. Silencing miR-144 in LMs reduces ROS release and leads to decreased expression of miR-144 in hepatocytes.

Fig. S4. Silencing miR-144 in LMs reduces oxidative stress and improves hepatic metabolism in insulin resistance.

Fig. S5. Proposed model of oxidative stress regulation by LMs in obesity.

Data file S1. Supplementary tables S1 to S9.

Table S1. GO biological processes (BP) enriched in genes differentially expressed between

9-week-old $o b / o b$ and wt mice based on RNA-seq.

Table S2. GO biological processes (BP) enriched in genes differentially expressed between 14-week-old $o b / o b$ and wt mice based on RNA-seq.

Table S3. Over-represented pathways between 9-week-old $o b / o b$ and wt mice based on RNA-seq.

Table S4. Over-represented pathways between 14-week-old ob/ob and wt mice based on RNA-seq.

Table S5. Enriched GO biological processes (BP) between HFD and ND mice based on GRO-seq. Table S6. Enriched GO biological processes (BP) between HFD- and ND-fed mice based on RNA-seq.

Table S7. Differentially expressed genes in HFD versus ND mice (GRO-seq) enriched in inflammatory response GO biological process (GO:0006954).

Table S8. Differentially expressed genes in HFD versus ND mice (RNA-seq) enriched in inflammatory response GO biological process (GO:0006954).

Table S9. Differential expression status of selected NRF2 target genes in 9-week-old $o b / o b$ and wt mice.

Data file S2. Raw data from figures.

References (54-68)

View/request a protocol for this paper from Bio-protocol.

\section{REFERENCES AND NOTES}

1. C. M. Hales, M. D. Carroll, C. D. Fryar, C. L. Ogden, Prevalence of obesity among adults and youth: United States, 2015-2016. NCHS Data Brief 2017, 1-8 (2017).

2. S. E. Kahn, R. L. Hull, K. M. Utzschneider, Mechanisms linking obesity to insulin resistance and type 2 diabetes. Nature 444, 840-846 (2006).

3. L. P. Bechmann, R. A. Hannivoort, G. Gerken, G. S. Hotamisligil, M. Trauner, A. Canbay, The interaction of hepatic lipid and glucose metabolism in liver diseases. J. Hepatol. 56, 952-964 (2012).

4. D. H. Ipsen, J. Lykkesfeldt, P. Tveden-Nyborg, Molecular mechanisms of hepatic lipid accumulation in non-alcoholic fatty liver disease. Cell. Mol. Life Sci. 75, 3313-3327 (2018).

5. Q. Liu, S. Bengmark, S. Qu, The role of hepatic fat accumulation in pathogenesis of non-alcoholic fatty liver disease (NAFLD). Lipids Health Dis. 9, 42 (2010).

6. J. Jager, M. Aparicio-Vergara, M. Aouadi, Liver innate immune cells and insulin resistance: the multiple facets of Kupffer cells. J. Intern. Med. 280, 209-220 (2016).

7. H.-Y. Tan, N. Wang, S. Li, M. Hong, X. Wang, Y. Feng, The reactive oxygen species in macrophage polarization: Reflecting its dual role in progression and treatment of human diseases. Oxid. Med. Cell. Longev. 2016, 2795090 (2016).

8. O. A. Castaneda, S.-C. Lee, C.-T. Ho, T.-C. Huang, Macrophages in oxidative stress and models to evaluate the antioxidant function of dietary natural compounds. J. Food Drug Anal. 25, 111-118 (2017).

9. L. Formentini, F. Santacatterina, C. Núñez de Arenas, K. Stamatakis, D. López-Martínez, A. Logan, M. Fresno, R. Smits, M. P. Murphy, J. M. Cuezva, Mitochondrial ROS production protects the intestine from inflammation through functional M2 macrophage polarization. Cell Rep. 19, 1202-1213 (2017).

10. C. Morgantini, J. Jager, X. Li, L. Levi, V. Azzimato, A. Sulen, E. Barreby, C. Xu, M. Tencerova, E. Näslund, C. Kumar, F. Verdeguer, S. Straniero, K. Hultenby, N. K. Björkström, E. Ellis, M. Rydén, C. Kutter, T. Hurrell, V. M. Lauschke, J. Boucher, A. Tomčala, G. Krejčová, A. Bajgar, M. Aouadi, Liver macrophages regulate systemic metabolism through non-inflammatory factors. Nat. Metab. 1, 445-459 (2019).

11. S. Vomund, A. Schäfer, M. J. Parnham, B. Brüne, A. Von Knethen, Nrf2, the master regulator of anti-oxidative responses. Int. J. Mol. Sci. 18, 2772 (2017).

12. S. Furukawa, T. Fujita, M. Shimabukuro, M. Iwaki, Y. Yamada, Y. Nakajima, O. Nakayama, M. Makishima, M. Matsuda, I. Shimomura, Increased oxidative stress in obesity and its impact on metabolic syndrome. J. Clin. Invest. 114, 1752-1761 (2004).

13. D. P. Bartel, MicroRNAs: Genomics, biogenesis, mechanism, and function. Cell 116, 281-297 (2004).

14. D. P. Bartel, MicroRNAs: Target recognition and regulatory functions. Cell 136, 215-233 (2009).

15. F. Ito, Y. Sono, T. Ito, Measurement and clinical significance of lipid peroxidation as a biomarker of oxidative stress: Oxidative stress in diabetes, atherosclerosis, and chronic inflammation. Antioxidants 8, 72 (2019).

16. K. Itoh, N. Wakabayashi, Y. Katoh, T. Ishii, T. O'Connor, M. Yamamoto, Keap1 regulates both cytoplasmic-nuclear shuttling and degradation of $\mathrm{Nrf2}$ in response to electrophiles. Genes Cells 8, 379-391 (2003). 
17. H. Dweep, N. Gretz, miRWalk2.0: A comprehensive atlas of microRNA-target interactions. Nat. Methods 12, 697 (2015).

18. P.-M. Ku, L.-J. Chen, J.-r. Liang, K.-C. Cheng, Y.-X. Li, J.-T. Cheng, Molecular role of GATA binding protein 4 (GATA-4) in hyperglycemia-induced reduction of cardiac contractility. Cardiovasc. Diabetol. 10, 57 (2011).

19. J. Jager, V. Corcelle, T. Grémeaux, K. Laurent, A. Waget, G. Pagès, B. Binétruy, Y. Le Marchand-Brustel, R. Burcelin, F. Bost, J. F. Tanti, Deficiency in the extracellular signal-regulated kinase 1 (ERK1) protects leptin-deficient mice from insulin resistance without affecting obesity. Diabetologia 54, 180-189 (2011).

20. M. Aouadi, G. J. Tesz, S. M. Nicoloro, M. Wang, M. Chouinard, E. Soto, G. R. Ostroff, M. P. Czech, Orally delivered siRNA targeting macrophage Map4k4 suppresses systemic inflammation. Nature 458, 1180-1184 (2009).

21. G. J. Tesz, M. Aouadi, M. Prot, S. M. Nicoloro, E. Boutet, S. U. Amano, A. Goller, M. Wang, C.-A. Guo, W. E. Salomon, J. V. Virbasius, R. A. Baum, M. J. O'Connor Jr., E. Soto, G. R. Ostroff, M. P. Czech, Glucan particles for selective delivery of siRNA to phagocytic cells in mice. Biochem. J. 436, 351-362 (2011).

22. C. C. Bell, V. M. Lauschke, S. U. Vorrink, H. Palmgren, R. Duffin, T. B. Andersson, M. Ingelman-Sundberg, Transcriptional, functional, and mechanistic comparisons of stem cell-derived hepatocytes, HepaRG cells, and three-dimensional human hepatocyte spheroids as predictive in vitro systems for drug-induced liver injury. Drug Metab. Dispos. 45, 419-429 (2017).

23. S. M. Shin, J. H. Yang, S. H. Ki, Role of the Nrf2-ARE pathway in liver diseases. Oxid. Med. Cell. Longev. 2013, 763257 (2013).

24. R. F. Schwabe, D. A. Brenner, Mechanisms of liver injury. I. TNF- $\alpha$-induced liver injury: Role of IKK, JNK, and ROS pathways. Am. J. Physiol. Gastrointest. Liver Physiol. 290, G583-G589 (2006).

25. R. Sano, J. C. Reed, ER stress-induced cell death mechanisms. Biochim. Biophys. Acta 1833, 3460-3470 (2013).

26. Y. Sumida, E. Niki, Y. Naito, T. Yoshikawa, Involvement of free radicals and oxidative stress in NAFLD/NASH. Free Radic. Res. 47, 869-880 (2013).

27. W. Tang, Y.-F. Jiang, M. Ponnusamy, M. Diallo, Role of Nrf2 in chronic liver disease. World J. Gastroenterol. 20, 13079-13087 (2014).

28. Q. Ma, Role of Nrf2 in oxidative stress and toxicity. Annu. Rev. Pharmacol. 53, 401-426 (2013).

29. M. McMahon, K. Itoh, M. Yamamoto, J. D. Hayes, Keap1-dependent proteasomal degradation of transcription factor Nrf2 contributes to the negative regulation of antioxidant response element-driven gene expression. J. Biol. Chem. 278, 21592-21600 (2003).

30. T. Nguyen, P. J. Sherratt, C. B. Pickett, Regulatory mechanisms controlling gene expression mediated by the antioxidant response element. Annu. Rev. Pharmacol. Toxicol. 43, 233-260 (2003).

31. E. L. Mills, D. G. Ryan, H. A. Prag, D. Dikovskaya, D. Menon, Z. Zaslona, M. P. Jedrychowski, A. S. H. Costa, M. Higgins, E. Hams, J. Szpyt, M. C. Runtsch, M. S. King, J. F. McGouran, R. Fischer, B. M. Kessler, A. F. McGettrick, M. M. Hughes, R. G. Carroll, L. M. Booty, E. V. Knatko, P. J. Meakin, M. L. J. Ashford, L. K. Modis, G. Brunori, D. C. Sévin, P. G. Fallon, S. T. Caldwell, E. R. S. Kunji, E. T. Chouchani, C. Frezza, A. T. Dinkova-Kostova, R. C. Hartley, M. P. Murphy, L. A. O'Neill, Itaconate is an anti-inflammatory metabolite that activates Nrf2 via alkylation of KEAP1. Nature 556, 113-117 (2018).

32. C. Sangokoya, M. J. Telen, J.-T. Chi, microRNA miR-144 modulates oxidative stress tolerance and associates with anemia severity in sickle cell disease. Blood 116, 4338-4348 (2010).

33. X. Zhang, X. Wang, H. Zhu, C. Zhu, Y. Wang, W. T. Pu, A. G. Jegga, G.-C. Fan, Synergistic effects of the GATA-4-mediated miR-144/451 cluster in protection against simulated ischemia/reperfusion-induced cardiomyocyte death. J. Mol. Cell. Cardiol. 49, 841-850 (2010).

34. T. Jourdan, J. K. Park, Z. V. Varga, J. Pálóczi, N. J. Coffey, A. Z. Rosenberg, G. Godlewski, R. Cinar, K. Mackie, P. Pacher, G. Kunos, Cannabinoid-1 receptor deletion in podocytes mitigates both glomerular and tubular dysfunction in a mouse model of diabetic nephropathy. Diabetes Obes. Metab. 20, 698-708 (2018).

35. M. Tencerova, M. Aouadi, P. Vangala, S. M. Nicoloro, J. C. Yawe, J. L. Cohen, Y. F. Shen L. Garcia-Menendez, D. J. Pedersen, K. Gallagher-Dorval, R. A. Perugini, O. T. Gupta, M. P. Czech, Activated Kupffer cells inhibit insulin sensitivity in obese mice. FASEB J. 29, 2959-2969 (2015).

36. G. Groeger, C. Quiney, T. G. Cotter, Hydrogen peroxide as a cell-survival signaling molecule. Antioxid. Redox Signal. 11, 2655-2671 (2009).

37. M. Reth, Hydrogen peroxide as second messenger in lymphocyte activation. Nat. Immunol. 3, 1129-1134 (2002).

38. E. McNeill, M. J. Crabtree, N. Sahgal, J. Patel, S. Chuaiphichai, A. J. Iqbal, A. B. Hale, D. R. Greaves, K. M. Channon, Regulation of iNOS function and cellular redox state by macrophage Gch1 reveals specific requirements for tetrahydrobiopterin in NRF2 activation. Free Radic. Biol. Med. 79, 206-216 (2015).

39. M. Mittal, M. R. Siddiqui, K. Tran, S. P. Reddy, A. B. Malik, Reactive oxygen species in inflammation and tissue injury. Antioxid. Redox Signal. 20, 1126-1167 (2014).
40. L. J. Hofseth, S. Saito, S. P. Hussain, M. G. Espey, K. M. Miranda, Y. Araki, C. Jhappan, Y. Higashimoto, P. He, S. P. Linke, M. M. Quezado, I. Zurer, V. Rotter, D. A. Wink, E. Appella, C. C. Harris, Nitric oxide-induced cellular stress and p53 activation in chronic inflammation. Proc. Natl. Acad. Sci. U.S.A. 100, 143-148 (2003).

41. J. Strom, B. Xu, X. Tian, Q. M. Chen, Nrf2 protects mitochondrial decay by oxidative stress. FASEB J. 30, 66-80 (2016).

42. M. Abdalkader, R. Lampinen, K. M. Kanninen, T. M. Malm, J. R. Liddell, Targeting Nrf2 to suppress ferroptosis and mitochondrial dysfunction in neurodegeneration. Front. Neurosci. 12, 466 (2018).

43. H. Zheng, S. A. Whitman, W. Wu, G. T. Wondrak, P. K. Wong, D. Fang, D. D. Zhang, Therapeutic potential of Nrf2 activators in streptozotocin-induced diabetic nephropathy. Diabetes 60, 3055-3066 (2011).

44. M. C. Jaramillo, D. D. Zhang, The emerging role of the Nrf2-Keap1 signaling pathway in cancer. Genes Dev. 27, 2179-2191 (2013).

45. H.-M. Ni, B. L. Woolbright, J. Williams, B. Copple, W. Cui, J. P. Luyendyk, H. Jaeschke, W.-X. Ding, Nrf2 promotes the development of fibrosis and tumorigenesis in mice with defective hepatic autophagy. J. Hepatol. 61, 617-625 (2014).

46. N. S. Rajasekaran, S. Varadharaj, G. D. Khanderao, C. J. Davidson, S. Kannan, M. A. Firpo, J. L. Zweier, I. J. Benjamin, Sustained activation of nuclear erythroid 2-related factor 2/ antioxidant response element signaling promotes reductive stress in the human mutant protein aggregation cardiomyopathy in mice. Antioxid. Redox Signal. 14, 957-971 (2011).

47. H. Wang, X. Liu, M. Long, Y. Huang, L. Zhang, R. Zhang, Y. Zheng, X. Liao, Y. Wang, Q. Liao, W. Li, Z. Tang, Q. Tong, X. Wang, F. Fang, M. Rojo de la Vega, Q. Ouyang, D. D. Zhang, S. Yu, H. Zheng, NRF2 activation by antioxidant antidiabetic agents accelerates tumor metastasis. Sci. Transl. Med. 8, 334ra351 (2016).

48. I. D. Podmore, H. R. Griffiths, K. E. Herbert, N. Mistry, P. Mistry, J. Lunec, Vitamin C exhibits pro-oxidant properties. Nature 392, 559 (1998).

49. E. R. Miller III, R. Pastor-Barriuso, D. Dalal, R. A. Riemersma, L. J. Appel, E. Guallar, Meta-analysis: High-dosage vitamin E supplementation may increase all-cause mortality. Ann. Intern. Med. 142, 37-46 (2005).

50. S. M. Lippman, E. A. Klein, P. J. Goodman, M. S. Lucia, I. M. Thompson, L. G. Ford, H. L. Parnes, L. M. Minasian, J. M. Gaziano, J. A. Hartline, J. K. Parsons, J. D. Bearden III, E. D. Crawford, G. E. Goodman, J. Claudio, E. Winquist, E. D. Cook, D. D. Karp, P. Walther, M. M. Lieber, A. R. Kristal, A. K. Darke, K. B. Arnold, P. A. Ganz, R. M. Santella, D. Albanes, P. R. Taylor, J. L. Probstfield, T. J. Jagpal, J. J. Crowley, F. L. Meyskens Jr., L. H. Baker, C. A. Coltman Jr., Effect of selenium and vitamin $E$ on risk of prostate cancer and other cancers: The selenium and vitamin E cancer prevention trial (SELECT). JAMA 301, 39-51 (2009).

51. C. C. Winterbourn, Reconciling the chemistry and biology of reactive oxygen species. Nat. Chem. Biol. 4, 278-286 (2008).

52. J.-H. Lee, D. Kim, H. J. Kim, C.-H. Lee, J. I. Yang, W. Kim, Y. J. Kim, J.-H. Yoon, S.-H. Cho, M.-W. Sung, H.-S. Lee, Hepatic steatosis index: A simple screening tool reflecting nonalcoholic fatty liver disease. Dig. Liver Dis. 42, 503-508 (2010).

53. B. Rosner, Fundamentals of Biostatistics (Belmont, CA: Thomson-Brooks/Cole, 2006)

54. M. Aparicio-Vergara, M. Tencerova, C. Morgantini, E. Barreby, M. Aouadi, Isolation of Kupffer cells and hepatocytes from a single mouse liver. Methods Mol. Biol. 1639, 161-171 (2017).

55. N. Akbar, J. E. Digby, T. J. Cahill, A. N. Tavare, A. L. Corbin, S. Saluja, S. Dawkins, L. Edgar, N. Rawlings, K. Ziberna, E. McNeill; Oxford Acute Myocardial Infarction (OxAMI) Study, E. Johnson, A. A. Aljabali, R. A. Dragovic, M. Rohling, T. G. Belgard, I. A. Udalova, D. R. Greaves, K. M. Channon, P. R. Riley, D. C. Anthony, R. P. Choudhury, Endotheliumderived extracellular vesicles promote splenic monocyte mobilization in myocardial infarction. JCI Insight 2, e93344 (2017).

56. B. Fang, L. J. Everett, J. Jager, E. Briggs, S. M. Armour, D. Feng, A. Roy, Z. Gerhart-Hines, Z. Sun, M. A. Lazar, Circadian enhancers coordinate multiple phases of rhythmic gene transcription in vivo. Cell 159, 1140-1152 (2014).

57. J. Wijkström, M. González-Quiroz, M. Hernandez, Z. Trujillo, K. Hultenby, A. Ring, M. Söderberg, A. Aragón, C.-G. Elinder, A. Wernerson, Renal morphology, clinical findings, and progression rate in mesoamerican nephropathy. Am. J. Kidney Dis. 69, 626-636 (2017).

58. M. C. Frith, U. Hansen, Z. Weng, Detection of cis -element clusters in higher eukaryotic DNA. Bioinformatics 17, 878-889 (2001).

59. D. Kim, G. Pertea, C. Trapnell, H. Pimentel, R. Kelley, S. L. Salzberg, TopHat2: Accurate alignment of transcriptomes in the presence of insertions, deletions and gene fusions. Genome Biol. 14, R36 (2013).

60. Y. Liao, G. K. Smyth, W. Shi, featureCounts: An efficient general purpose program for assigning sequence reads to genomic features. Bioinformatics 30, 923-930 (2014).

61. R. Quinlan, I. M. Hall, BEDTools: A flexible suite of utilities for comparing genomic features. Bioinformatics 26, 841-842 (2010).

62. A. Dobin, C. A. Davis, F. Schlesinger, J. Drenkow, C. Zaleski, S. Jha, P. Batut, M. Chaisson, T. R. Gingeras, STAR: Ultrafast universal RNA-seq aligner. Bioinformatics 29, 15-21 (2013).

63. C. Trapnell, B. A. Williams, G. Pertea, A. Mortazavi, G. Kwan, M. J. van Baren, S. L. Salzberg, B. J. Wold, L. Pachter, Transcript assembly and quantification by RNA-Seq reveals 
unannotated transcripts and isoform switching during cell differentiation. Nat. Biotechnol. 28, 511-515 (2010)

64. C. Trapnell, D. G. Hendrickson, M. Sauvageau, L. Goff, J. L. Rinn, L. Pachter, Differential analysis of gene regulation at transcript resolution with RNA-seq. Nat. Biotechnol. 31, 46-53 (2013).

65. D. N. Slenter, M. Kutmon, K. Hanspers, A. Riutta, J. Windsor, N. Nunes, J. Mélius, E. Cirillo, S. L. Coort, D. Digles, F. Ehrhart, P. Giesbertz, M. Kalafati, M. Martens, R. Miller, K. Nishida, L. Rieswijk, A. Waagmeester, L. M. T. Eijssen, C. T. Evelo, A. R. Pico, E. L. Willighagen, WikiPathways: A multifaceted pathway database bridging metabolomics to other omics research. Nucleic Acids Res. 46, D661-D667 (2018).

66. M. Martin, Cutadapt removes adapter sequences from high-throughput sequencing reads. EMBnet.J. 17, 10-12 (2011).

67. M. J. Axtell, ShortStack: Comprehensive annotation and quantification of small RNA genes. RNA 19, 740-751 (2013).

68. J. Ye, S. McGinnis, T. L. Madden, BLAST: Improvements for better sequence analysis. Nucleic Acids Res. 34, W6-W9 (2006).

Acknowledgments: We are grateful to R. Harris for input on the manuscript. Funding: This work was supported by research grants from AstraZeneca through the ICMC (M.A.), the Swedish Research Council (M.A.: 2015-0358), Stockholm County Council (M.R.), the Novo Nordisk Foundation, including the Tripartite Immunometabolism Consortium (M.A. and M.R. TrIC; NNF15CC0018486), the Strategic Research Program in Diabetes at Karolinska Institutet (M.A. and M.R.), The Erling-Persson Family Foundation (A.T.), and the Diabetes Wellness Foundation Sweden (J.J.). Author contributions: V.A., J.J., and P.C. carried out most experiments. C.M., L.L., E.B., A.S., J.W., C.O., and C.X. helped with hepatocyte and LM isolation, flow cytometry, and interpretation of the data. J.X.S. and V.M.L. provided and performed liver spheroid treatments. N.A. and R.P.C. performed EV experiments. L.H. generated all electron microscopy images and measurements. E.E. provided biopsies and liver cells from lean individuals. E.N., A.T., E.E., K.W., and M.R. provided human liver biopsies. P.C. and X.L. performed bioinformatics analysis and interpretation. V.A., J.J., and M.A. conceived the project, analyzed data, and wrote the manuscript with input from all coauthors. S.M.C. helped with interpretation of the data and contributed to writing the revised manuscript. Competing interests: V.A. and M.A. are inventors of a pending filing patent application (UK1910299.5, "Medical uses, methods, and uses") through Potter Clarkson LLP. V.M.L. is cofounder and shareholder of HepaPredict AB. Data and materials availability: All data associated with this study are present in the paper or the Supplementary Materials. Gene expression sequencing reads and data from this study are available from the Gene Expression Omnibus database under accessions GSE132795, GSE132800, and GSE132801.

Submitted 11 February 2019

Accepted 6 February 2020

Published 26 February 2020

10.1126/scitranslmed.aaw9709

Citation: V. Azzimato, J. Jager, P. Chen, C. Morgantini, L. Levi, E. Barreby, A. Sulen, C. Oses, J. Willerbrords, C. Xu, X. Li, J. X. Shen, N. Akbar, L. Haag, E. Ellis, K. Wålhen, E. Näslund, A. Thorell, R. P. Choudhury, V. M. Lauschke, M. Rydén, S. M. Craige, M. Aouadi, Liver macrophages inhibit the endogenous antioxidant response in obesity-associated insulin resistance. Sci. Transl. Med. 12, eaaw9709 (2020). 


\section{Science Translational Medicine}

\section{Liver macrophages inhibit the endogenous antioxidant response in obesity-associated insulin resistance}

Valerio Azzimato, Jennifer Jager, Ping Chen, Cecilia Morgantini, Laura Levi, Emelie Barreby, André Sulen, Carolina Oses, Joost Willerbrords, Connie Xu, Xidan Li, Joanne X. Shen, Naveed Akbar, Lars Haag, Ewa Ellis, Kerstin Wålhen, Erik Näslund, Anders Thorell, Robin P. Choudhury, Volker M. Lauschke, Mikael Rydén, Siobhan M. Craige and Myriam Aouadi

Sci Transl Med 12, eaaw9709.

DOI: 10.1126/scitranslmed.aaw9709

Muted macrophages

Obesity and impaired insulin sensitivity are known to predispose to nonalcoholic fatty liver disease. Azzimato et al. show that liver macrophages (LMs) contribute to a weakened antioxidant response to hepatic lipid accumulation in $o b / o b$ mice fed a high-fat diet. LMs in the metabolically dysregulated animals showed increased transcription of miR-144, a microRNA that impaired the LM antioxidant response by targeting nuclear factor erythroid 2-related factor 2 (NRF2). Targeting this microRNA restored the LM response to oxidative damage in human 3D liver cell culture and improved insulin resistance in obese mice. miR-144 was also up-regulated in obese insulin-resistant humans, suggesting the potential clinical relevance of the mechanism.

ARTICLE TOOLS

SUPPLEMENTARY MATERIALS

RELATED

CONTENT

REFERENCES

PERMISSIONS http://stm.sciencemag.org/content/12/532/eaaw9709

http://stm.sciencemag.org/content/suppl/2020/02/24/12.532.eaaw9709.DC1 http://stm.sciencemag.org/content/scitransmed/11/512/eaay0284.full http://stm.sciencemag.org/content/scitransmed/11/520/eaav9701.full http://stm.sciencemag.org/content/scitransmed/10/461/eaar2680.full http://stm.sciencemag.org/content/scitransmed/10/454/eaan1230.full

This article cites 67 articles, 8 of which you can access for free http://stm.sciencemag.org/content/12/532/eaaw9709\#BIBL

http://www.sciencemag.org/help/reprints-and-permissions

Science Translational Medicine (ISSN 1946-6242) is published by the American Association for the Advancement of Science, 1200 New York Avenue NW, Washington, DC 20005. The title Science Translational Medicine is a registered trademark of AAAS.

Copyright (C 2020 The Authors, some rights reserved; exclusive licensee American Association for the Advancement of Science. No claim to original U.S. Government Works 